\title{
DUAL SPACES OF GROUPS WITH PRECOMPACT CONJUGACY CLASSES
}

BY

\author{
JOHN R. LIUKKONEN
}

\begin{abstract}
We show that a second countable locally compact type I group with a compact invariant neighborhood of the identity is CCR, and has a Hausdorff dual if and only if its conjugacy classes are precompact. We obtain sharper results if the group is almost connected or has a fundamental system of invariant neighborhoods of the identity. Along the way we show that for a locally compact abelian group $A$ and a group $B$ of topological group automorphisms of $A, A$ has small $B$ invariant neighborhoods at 1 if and only if $\hat{A}$ has precompact orbits under the dual actions of $B$.
\end{abstract}

1. Introduction and preliminaries. One of the striking features of the Fell topology on the dual space $\hat{G}$ of the locally compact group $G$ is its characterization of important properties of $G$ by simple separation properties of $\hat{G}$. Thus for second countable $G, G$ is type $I$ if and only if $\hat{G}$ is $T_{0}$ and CCR if and only if $\hat{G}$ is $T_{1}$. Moreover, Baggett $[2]$ has recently shown that, for second countable $G$, $\hat{G}$ discrete is equivalent to $G$ compact. One is naturally led to ask what significance, if any, a Hausdorff dual space has, and while many of our results do not pertain to this question, it is nonetheless the primary motivation of this paper. Our principal results are that a type $I[I N]$ group is CCR and has Hausdorff dual if and only if it is $[F C]^{-}$; we obtain more explicit results in the case of an [SIN] group or an almost connected group.

Most of this paper is taken from the author's doctoral dissertation, submitted to Columbia University in June, 1970. I thank my adviser, Martin Moskowitz, for his advice and encouragement during the time in which this research was carried out. I would also like to thank Richard Mosak for many stimulating discussions concerning harmonic analysis.

We now establish some conventions and notation. Groups $G$ will always be second countable locally compact, except in $\$ 2$. Representations of groups will be strongly continuous unitary representations, and representations of $C^{*}$ algebras will be nondegenerate ${ }^{*}$ representations (hence continuous). The letters $\mathbf{R}, \mathbf{Z}$ and $T$ will denote the real numbers, the integers, and the circle; the circle will

Received by the editors June 4, 1971 and, in revised form, March 7, 1972.

AMS (MOS) subject classifications (1970). Primary 22D05, 22D10, 22D35; Secondary 22B99.

Key words and phrases. $[F C]^{-}$group, $[I N]$ group, $[S I N]$ group; $[Z]$ group, Fell dual space, Hausdorff dual, type I, CCR. 
be regarded as the complex numbers of modulus one under multiplication. We record here some important classes of groups together with their defining properties: $[F C]^{-}$(having precompact conjugacy classes), $[F D]^{-}$(having a precompact commutator subgroup), [IN] (having a compact neighborhood of 1 invariant under inner automorphisms), [SIN] (having a fundamental system of invariant neighborhoods at 1 ), and $[Z]$ (being compact modulo the center). The following inclusions hold: $[Z] \subset[S I N] \subset[I N]$ and $[Z] \subset[F D]^{-} \subset[F C]^{-} \subset[I N]$. (See [12] and [25].)

An exact sequence of locally compact groups will be an algebraic exact sequence $(1) \rightarrow G \rightarrow H \rightarrow J \rightarrow(1)$ in which the maps are continuous and open. Since we are taking groups to be second countable, it will be enough to have the maps continuous.

The continuous complex valued function $f$ on $G$ will be called central if $f(\alpha x)=f(x)$ for all $x \in G$ and all inner automorphisms $\alpha$. Notice that if the automorphism $\beta$ is the point open limit of inner automorphisms then $f(\beta x)=f(x)$ for all $x \in G$. The continuous functional $F$ on the Banach algebra $A$ will be called central if $F(a b)=F(b a)$ for all $a, b, \in A$.

We will take 2 cocycles of $G$ to be those with coefficients in $T$, where $G$ acts trivially on $T$. We note that if $\alpha$ is a 2 cocycle of $G, \alpha$ defines a central exact sequence $(1) \rightarrow T \rightarrow H \rightarrow G \rightarrow(1)$ of locally compact groups, and a representations of $G$ are in one to one correspondence with ordinary representations of $H$ whose restrictions to $T$ are multiples of the character $t \mapsto t$. We will denote the irreducible $a$ representations of $G$ by ${ }_{a} \hat{G}$ and the image of $Z(H)$ (the center of $H)$ in $G$ by $Z_{a}(G)$. For details and further references on 2 cocycles and their relation to representation theory, we refer the reader to Chapter I of [1]. The same source contains a sketch of the Mackey theory of induced representations, which we will use extensively.

1.1 Proposition. Let $\Phi$ be the continuous positive definite functions $\phi$ of $G$ with $\phi(e) \leq 1$. Let $\mathcal{F}$ be the positive functionals on $L^{1}(G)$ of norm $\leq 1$. Then there is a bijection between $\Phi$ and $\mathcal{F}$ given by $F(\phi)(f)=\int f(x) \phi(x) d x$ for all $f \in L^{1}(G)$. Moreover central elements $\Phi_{c}$ of $\Phi$ correspond to central elements $\mathcal{F}_{c}$ of $\mathcal{F}$ under this bijection and $\Phi$ and $\Phi_{c}$ are compact metric in the weak ${ }^{*}$ topology inberited from $\mathcal{F}$ and $\mathcal{F}_{c}$.

Proof. The first assertion is part of Théorème 13.4.5 of [5]. Verifying that $\Phi_{c}$ and $\mathcal{F}_{c}$ correspond under the bijection is completely straightforward. It is clear that $\mathcal{F}^{\mathcal{F}}$ and $\mathcal{F}_{c}$ are weak ${ }^{*}$ closed in the unit ball of $L^{\infty}(G)$, and finally, it is well known that the unit ball of the dual space of a separable Banach space is compact metric in the weak ${ }^{*}$ topology (see for example B7 of [5]). 
1.2 Definition. We denote by $\mathbb{Q}(G)$ the group of topological group automorphisms of $G$. The topology on $\mathbb{Q}(G)$ (see [4]) is given as follows: $\alpha_{\nu} \rightarrow \alpha$ in $\mathbb{A}(G)$ if and only if $\alpha_{\nu} \rightarrow \alpha$ and $\alpha_{\nu}^{-1} \rightarrow \alpha^{-1}$ both uniformly on compacta. One may verify that the evaluation map $\mathscr{Q}(G) \times G \rightarrow G$ is jointly continuous (see p. 40 of Hochschild, The structure of Lie groups). We let $I(G)$ be the closure in $\mathbb{Q}(G)$ of the group of inner automorphisms.

1.3 Proposition. $I(G)$ is compact if and only if $G \in[F C]^{-} \cap[S I N]$.

This is Theorem 4.1 of $[10]$ in the special case that $B^{-}=I(G)$. In general one has that the subgroup $B$ of $\mathbb{Q}(G)$ is precompact iff the $B$ orbits of $G$ are precompact and the identity of $G$ has a fundamental system of $B$ invariant neighborhoods; moreover, one need not assume $G$ is separable.

1.4 Definition. If $f$ is a continuous complex valued function on $G$ and $K$ is a compact subset of $G$, let $\|f\|_{K}=\sup \{|f(x)| \mid x \in K\}$. Let $G \in[F C]^{-} \cap[S I N]$. Then $f(\alpha x)$ is continuous and bounded in $\alpha \in I(G)$ for each fixed $x$ and we may define the function $f^{\#}$ by $f^{\#}(x)=\int_{I(G)} f(a x) d \alpha$, where $d \alpha$ is normalized Haar measure on the compact group $I(G)$.

1.5 Proposition. Suppose $f$ is a continuous complex valued function on the $[F C]^{-} \cap[S I N]$ group G. Then $f^{\#}$ is continuous and central. If $f$ is central, $f^{\#}=f$. If $f$ is positive definite, so is $f^{\#}$. Moreover, $\left\|f^{\#}\right\|_{K} \leq\|f\|_{I(G)(K)}$, where $K$ is any compactum in $G$ and $I(G)(K)$ is the compact image of $I(G) \times K$ under the evaluation map. Finally, if $f$ has compact support, then so does $f^{\#}$.

Proof. Straightforward verification. See [21], for example.

1.6 Proposition. Suppose $G \in[F C]^{-} \cap[S I N]$. Let $\pi$ be a continuous finite dimensional irreducible representation of $G, v$ a unit vector in $H_{\pi}$, and let $\phi(x)=\left(\pi_{x} v, v\right)$. Then $\phi^{\#}(x)=(\operatorname{deg} \pi)^{-1} \operatorname{Tr} \pi_{x}$ for all $x \in G$.

Proof. First we note that by $1.1, F\left(\phi^{\sharp}\right)$ is a central functional on $L^{1}(G)$. Suppose now that $f \in \operatorname{ker} \pi$; that is, $\int f(x) \pi_{x} d x=0$ where $f \in L^{1}(G)$. Then for each $y \in G, \int f(x)_{y x y^{-1}} d x=\pi_{y} \int f(x)_{x} d x \pi_{y^{-1}}=0$. Moreover if $a \in I(G)$, we have inner automorphisms $\alpha_{n} \rightarrow a$ and so for each $u, v \in H_{\pi}$ we have

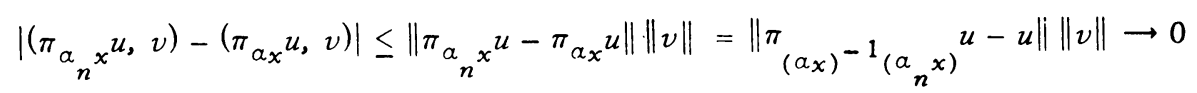

uniformly on $G$ compacta because $a_{n} \rightarrow a$ uniformly on $G$ compacta. It follows that $\int f(x)\left(\pi_{a x} u, v\right) d x=0$ for all $u, v$ and furthermore that

$$
\int f(x) \phi^{\sharp}(x) d x=\int_{I(G)} \int_{G} f(x)\left(\pi_{a x} v, v\right) d x d \alpha=0 .
$$


Thus $F\left(\phi^{\sharp}\right)$ defines a positive central functional on the image of $L^{1}(G)$ under $\pi$. Since $\pi$ is irreducible this image is a full matrix algebra $B\left(H_{\pi}\right)$ and so $F\left(\phi^{\#}\right)(f)=$ $\lambda \operatorname{Tr} \pi(f)$ for all $f \in L^{1}(G)$, for some constant $\lambda$. It follows easily that $\phi^{\#}(x)=$ $\lambda \operatorname{Tr} \pi_{x}$ for all $x \in G$ and, since $\phi^{\#}(1)=1, \lambda=(\operatorname{deg} \pi)^{-1}$ and the proposition is proved.

1.7 Proposition. Let $G$ be locally compact abelian, and let a $\in H^{2}(G, T)$. Then for any cocycle a representing $a, \alpha^{(2)}(x, y)=\alpha(x, y) \alpha(y, x)^{-1}$ is jointly continuous and bilinear (a character in each variable separately). Moreover, $\alpha^{(2)}$ is independent of the choice of $\alpha$. Finally, we may choose a so that $\alpha(x, y)=1$ whenever $x, y$ are in $Z_{\alpha}(G)=\left\{w \in G \mid \alpha^{(2)}(w, v)=1\right.$ for all $v$ in $\left.G\right\}$.

Proof. The first assertion is Lemma 7.1 of [1.6] and the second is Lemma 7.2 of [16]. Let $\beta$ be a cocycle representing $a$. Then there is a unique locally compact group $H$ which as a Borel group is $T \times G$ with multiplication defined by $\langle s, x\rangle\langle t, y\rangle=\langle s t \beta(x, y), x+y\rangle$ for $s, t \in T$ and $x, y \in G$. Of course $(1) \rightarrow T \rightarrow$ $H \stackrel{\pi}{\rightarrow} G \rightarrow(1)$ (up to equivalence of extensions) is the extension defined by $a$. Since $Z(H) \supset T$, we have $Z(H)=T \oplus A$ for some closed subgroup $A$ of $Z(H)$. Moreover, $\pi: A \rightarrow \pi(Z(H))$ is a topological group isomorphism. Define a Borel cross-section $\sigma: G \rightarrow H$ as follows: $\sigma(x)=(\pi \mid A)^{-1}(x)$ if $x \in \pi(Z(H))$ and $\sigma(x)=$ $\langle 0, x\rangle$ if $x \notin \pi(Z(H))$. (To see that this is Borel use Lemma 1.2 of [17].) Let $\alpha(x, y)=\sigma(x) \sigma(y) \sigma(x+y)^{-1}$ for all $x, y \in G$. We then have $\alpha$ cohomologous to $\beta$ (see discussion on Pp. 24-25 of [1]) and since $\sigma \mid \pi(Z(H))$ is a group homomorphism, $\alpha$ is trivial on $\pi(Z(H))=Z_{\alpha}(G)$. This proves the proposition.

2. Automorphisms of $[F C]^{-}$groups. In this section we prove a result on automorphisms of $[F C]^{-}$groups which leads to a result of Robertson [25] and an additional application. In this section only, $G$ will not be assumed second countable. Recall that a periodic group (see [12] for example) is one each element of which is contained in a compact subgroup.

2.1 Proposition. Let $P$ be a locally compact periodic group whose $B$ orbits are precompact, where $B \subset \mathfrak{Q}(G)$ is a subgroup containing the inner automorphisms. Then $P$ contains a compact open $B$ invariant subgroup $K$.

Proof. Let $N$ be a compact neighborhood of 1 in $P$. According to Theorem 3.11 of [12], $N$ generates a compact open subgroup $K_{1}$ of $P$. We assume first that $K_{1}$ has countable index in $P$, and let $x_{1}=1, x_{2}, x_{3}, \cdots$ be a set of coset representatives for $K_{1}$ in $P$. A set $S$ is precompact in $P$ if and only if $S \subset$ $\bigcup_{i=1}^{n} x_{i} K_{1}$ for some integer $n$. Now for each $n \geq 1$, let $S_{n}$ be the closed set

$$
K_{1} \cap \bigcap_{a \in B} \alpha^{-1}\left(\bigcup_{i=1}^{n} x_{i} K_{1}\right)=\left\{x \in K_{1} \mid B(x) \subset \bigcup_{i=1}^{n} x_{i} K_{1}\right\} .
$$


Since each element of $K_{1}$ has a precompact orbit under $B, K_{1}=\bigcup_{n=1}^{\infty} S_{n}$. There fore by the Baire category theorem some $S_{n}$ contains an open set $U$. Now $B\left(S_{n}\right) C$ $\mathcal{U}_{i=1}^{n} x_{i} K_{1}$. Therefore if $K$ is the subgroup generated by $B\left(S_{n}\right)$ and $K_{2}$ is the subgroup generated by $\bigcup_{i=1}^{n} x_{i} K_{1}$, we have $K$ open, $K_{2}$ compact (by 3.11 of [12]) and $K \subset K_{2}$. Moreover, $B\left(B\left(S_{n}\right)\right)=B\left(s_{n}\right)$, and so $K$ is $B$ invariant.

Now let us drop the assumption that $K_{1}$ has countable index in $P$, and suppose that $B\left(K_{1}\right)$ is not precompact. In particular suppose there is a countably infinite set $x_{1}, x_{2}, \cdots$ of coset representatives of $K_{1}$ in $P$ such that, for each $i$, there is a $\beta_{i} \in B$ with $\beta_{i}\left(K_{1}\right) \cap x_{i} K_{1} \neq \varnothing$. If $B^{\prime}$ is the countable subgroup of $B$ generated by $\beta_{1}, \beta_{2}, \cdots$, then $B^{\prime}\left(K_{1}\right)$ is not precompact. Let $H$ be the open subgroup of $P$ generated by $B^{\prime}\left(K_{1}\right)$. We claim that $\left[H: K_{1}\right]$ is countable. In fact, for $\beta_{1}^{\prime}, \cdots, \beta_{n}^{\prime} \in B^{\prime}$ let $K\left(\beta_{1}^{\prime}, \cdots, \beta_{n}^{\prime}\right)$ be the group generated by $K_{1}, \beta_{1}^{\prime}\left(K_{1}\right), \ldots$, $\beta_{n}^{\prime}\left(K_{n}\right)$. Then by 3.11 of [12] we know $K\left(\beta_{1}^{\prime}, \cdots, \beta_{n}^{\prime}\right)$ is compact, and since $K_{1}$ is open we have the homogeneous space $K\left(\beta_{1}^{\prime}, \ldots, \beta_{n}^{\prime}\right) / K_{1}$ finite.

Since each element of $H$ is contained in some $K\left(\beta_{1}^{\prime}, \ldots, \beta_{n}^{\prime}\right)$ and there are only countably many choices of $\beta_{1}^{\prime}, \cdots, \beta_{n}^{\prime}$ from $B^{\prime}$ possible, we must have that $H$ is contained in countably many $K_{1}$ cosets.

Now let $B^{\prime \prime}$ be the subgroup of $B$ generated by $B^{\prime}$ and the inner automorphisms corresponding to elements of $H$. Certainly $H$ is $B^{\prime \prime}$ invariant and has precompact $B^{\prime \prime}$ orbits; moreover $H$ is periodic and contains a compact open subgroup $K_{1}$ of countable index. By the reasoning in the first paragraph, $H$ contains a $B^{\prime \prime}$ invariant compact open subgroup $K$. The index $\left[K_{1}: K \cap K_{1}\right]<\infty$ and so we have coset representatives $y_{1}, \cdots, y_{n}$ for $K \cap K_{1}$ in $K_{1}$. Thus

$$
B^{\prime}\left(K_{1}\right)=B^{\prime}\left(\bigcup_{y_{i}}\left(K \cap K_{1}\right)\right)=\bigcup^{\prime}{ }^{\prime}\left(y_{i}\right) B^{\prime}\left(K \cap K_{1}\right) \subset\left[\bigcup_{B^{\prime}}\left(y_{i}\right)\right] K .
$$

Each $B^{\prime}\left(y_{i}\right)$ is precompact, however, and so $B^{\prime}\left(K_{1}\right)$ is precompact, which is impossible by the construction of $B^{\prime}$. This contradiction proves that $B\left(K_{1}\right)$ is precompact. By 3.11 of [12], $B\left(K_{1}\right)$ generates a compact open subgroup of $P$ which is clearly $B$ invariant.

The following is a slight extension of a result due to Robertson [25]. We include a proof for completeness.

2.2 Theorem. Suppose that $G$ has precompact $B$ orbits, where $B \subset \mathbb{Q}(G)$ is a subgroup containing the inner automorphisms. Then $G$ satisfies an exact sequence $(1) \rightarrow K \rightarrow G \rightarrow \mathbf{R}^{n} \times D \rightarrow(1)$, where $K$ is compact $B$ invariant, $D$ is discrete $F C$, and $\mathbf{R}^{n} \times D$ bas precompact orbits under the group $B^{\prime}$ of induced actions of $B$. Furtbermore $\mathbf{R}^{n}$ is $B^{\prime}$ invariant and bas small $B^{\prime}$ invariant neigh. borboods of the identity.

Before proving 2.2 we state two additional results we require. 
2.3 Theorem. Suppose that $G$ bas precompact $B$ orbits where $B \subset \mathbb{Q}(G)$ is a subgroup including the inner automorphisms. Then $G$ satisfies an exact sequence $(1) \rightarrow P \rightarrow G \rightarrow \mathbf{R}^{n} \times D \rightarrow(1)$, where $P$ is the closed characteristic subgroup of periodic elements and $D$ is discrete torsion free abelian.

Proof. This is most of Theorem 3.16 of [12].

2.4 Proposition. Suppose (1) $\rightarrow P \rightarrow G \rightarrow \mathbf{R}^{n} \times D \rightarrow(1)$ is an exact sequence of locally compact groups, where $P$ is a discrete torsion group and $D$ is discrete torsion free abelian. If $G \in[F C]^{-}$, then $G \cong \mathbf{R}^{n} \times H$, where $H$ is discrete $F C$.

Proof of 2.4. Clearly $G$ is locally euclidean and is thus a Lie group. By Theorem 3.20 of [12], the identity component $G_{0}$ of $G$ has precompact commutator subgroup; moreover the closed commutator of $G_{0}$ is connected, and the abelian quotient of $G_{0}$ by its closed commutator is of the form $\mathbf{R}^{m} \times T^{p}$. Hence $G_{0}$ contains a compact connected, characteristic subgroup $K_{1}$ such that $G_{0} / K_{1}$ is a vector group. Thus the periodic elements $P \cap G_{0}$ of $G_{0}$ are connected, and since $P$ is discrete in $G$, we therefore have $P \cap G_{0}=(1)$. We also have that the image of $G_{0}$ in $G / P$ is an analytic subgroup of $\mathbf{R}^{n}$ and is therfore a closed connected subgroup of $\mathbf{R}^{n}$; it is easy to see that the image of $G_{0}$ is all of $\mathbf{R}^{n}$ and in fact the map $G \rightarrow \mathbf{R}^{n} \times D$ defines a topological group isomorphism of $G_{0}$ onto $\mathbf{R}^{n}$. Now define $H$ as the preimage of $D$ in $G$. Clearly $G_{0} \cap H \subset P$, so that $G_{0} \cap H \subset G_{0} \cap P=(1)$. Moreover $H$ is discrete, and therefore the connected subgroup $G_{0}$ acts trivially by inner automorphisms on each element of $H$. Thus $G_{0}$ and $H$ commute and since $G=G_{0} H$, we have $G=G_{0} \times H \cong \mathbf{R}^{n} \times H$. Finally $H$ as the discrete quotient of an $[F C]^{-}$group is itself $F C$.

Proof of 2.2. Since $G$ has precompact $B$ orbits, Theorem 2.3 implies that $G$ satisfies the exact sequence $(1) \rightarrow \mathrm{P} \rightarrow G \rightarrow \mathrm{R}^{n} \times D^{\prime} \rightarrow(1)$ where $P$ is the group of periodic elements and $D^{\prime}$ is discrete torsion free abelian. By $2.1, P$ contains a compact open $B$ invariant subgroup $K$, and thus $G / K$ satisfies the exact sequence $(1) \rightarrow P / K \rightarrow G / K \rightarrow \mathbf{R}^{n} \times D^{\prime} \rightarrow(1)$ where $P / K$ is a discrete torsion group and $G / K$ has precompact $B^{\prime}$ orbits, where $B^{\prime}$ is the group of actions induced in $G / K$ by $B$. Thus by 2.4 we have $G / K \cong \mathbf{R}^{n} \times D$ where $D$ is discrete $F C$. Since $\mathbf{R}^{n}$ is the identity component of $G / K$ it is invariant under $B^{\prime}$. Since $\mathbf{R}^{n}$ has precompact $B^{\prime}$ orbits it has small $B^{\prime}$ invariant neighborhoods at 1 (see 2.6). This proves 2.2 .

Corollary to 2.2. For any locally compact group $G$ let $G_{F}$ denote the normal subgroup of elements with precompact conjugacy classes. Then $G \in[I N]$ if and only if $G_{F}$ is open. (See also [29].)

Proof. If $G \in[I N]$ then $G$ has a compact invariant neighborhood of 1 which 
is clearly contained in $G_{F}$. Thus $G_{F}$ is open. If on the other hand $G_{F}$ is open, then by 2.2 we have an exact sequence (1) $\rightarrow K \rightarrow G_{F} \rightarrow \mathbf{R}^{n} \times D \rightarrow(1)$ where $K$ is normal in $G$ and $\mathbf{R}^{n}$ has a compact neighborhood of the identity invariant under the inner automorphisms of $G$. The preimage of this neighborhood in $G_{F}$ is a compact invariant neighborhood of 1 in $G$.

As another application of 2.1 , we show that, for a locally compact abelian $G$ and a group $B$ of automorphisms of $G, G$ has small $B$ invariant neighborhoods if and only if $\hat{G}$ has precompact $B^{\wedge}$ orbits. Here $B^{\wedge}$ is the group of dual automorphisms to $B$ acting on $\hat{G}$. We believe that this result sheds some light on why the $[S I N]$ condition is needed in 1.3 .

2.5 Proposition. Suppose $G$ is locally compact abelian and $B$ is a group of automorphisms of $G$ such that $G$ bas small $B$ invariant neighborboods. Then $\hat{G}$ bas precompact $B^{\wedge}$ orbits.

Proof. Suppose that $G$ has small $B$ invariant neighborhoods; let $\epsilon>0$ and let $\chi \in \hat{G}$. Let $U$ be a $B$ invariant neighborhood of 1 in $G$ such that $|\chi(x)-\chi(1)|<\epsilon$ for all $x \in U$. Then $|(\chi \circ \beta)(x+y)-(\chi \circ \beta)(y)|=$ $|\chi(\beta x)-\chi(1)|<\epsilon$ for all $x \in U$ and $\beta \in B$, for each $y \in G$. It follows that the compact open closure $\bar{S}$ of the set $S=\{\chi \circ \beta \mid \beta \in B\}$ is equicontinuous at each point of $G$. Of course range $f \subset T$ for each $f \in \bar{S}$ and it follows by the Ascoli Theorem, p. 233 of [15], that $\bar{S}$ is compact. Since $\chi$ is arbitrary this shows that $\hat{G}$ has precompact $B \hat{}$ orbits.

2.6. Lemma. If $K$ is compact abelian and $\hat{K}$ bas finite $B^{\wedge}$ orbits (wbere $B \subset \mathbb{Q}(K)$ is a subgroup) then $K$ bas small $B$ invariant neighborboods. If on the other band $B$ is a group of automorphisms of $\mathbf{R}^{n}$ such that $\mathbf{R}^{n}$ bas precompact $B$ orbits, then $\mathbf{R}^{n}$ bas small $B$ invariant neighborboods and it follows that $\hat{\mathbf{R}}^{n}$ bas precompact $B^{\wedge}$ orbits and small $B^{\wedge}$ invariant neighborboods.

Proof. First let $B$ be a group of automorphisms of the compact abelian group $K$ such that $\hat{K}$ has finite $B^{\wedge}$ orbits. Let $U$ be a neighborhood of 1 in $K$. Then there are $\chi_{1}, \cdots, \chi_{n}$ in $\hat{K}$ and $\epsilon>0$ such that

$$
\begin{aligned}
W & =W\left(\chi_{1}, \cdots, \chi_{n}, \epsilon\right) \\
& =\left\{x \in K|| \chi_{i}(x)-1 \mid<\epsilon \text { for } i=1,2, \cdots, n\right\} \subset U .
\end{aligned}
$$

This last assertion follows from the fact that $\hat{K}$ is discrete and so $W\left(\chi_{1}, \cdots, \chi_{n}, \epsilon\right)$ defines a compact open neighborhood of 1 in $K$ regarded as the dual of $\hat{K}$. Now each $\chi_{i}$ has a finite $B$ orbit; therefore there is a subgroup $B_{1}$ of finite index in $B$ such that $\left(B_{1}\right)$ leaves $\chi_{1}, \cdots, \chi_{n}$ fixed. Then $B_{1}$ leaves $W$ invariant and if $b_{1}, \ldots, b_{n}$ are coset representatives for $B_{1}$ in $B$ we have that $\bigcap_{b \in B} b W=\bigcap_{i=1}^{n} b_{i} W \subset U$ is a $B$ invariant neighborhood. This proves the 
first assertion. The assertion about $R^{n}$ is well known and can be proved by showing $B$ is precompact in $\operatorname{GL}(n, \mathbf{R})$ and then using the Weyl unitary trick to show $B$ can be regarded as a group of orthogonal operators on $\mathbf{R}^{n}$.

The compact case of the following theorem was used in 4.11 of [12].

2.7 Theorem. Suppose $G$ is locally compact abelian and $B$ is a group of automorphisms of $G$. Then $G$ bas small $B$ invariant neighborboods if and only if $\hat{G}$ has precompact $B^{\wedge}$ orbits.

Proof. The "only if " part is 2.5. Therefore suppose $\hat{G}$ has precompact $B$ orbits. We may write $G=\mathbf{R}^{n} \times H$ and $\hat{G}=\hat{\mathbf{R}}^{n} \times \hat{H}$, where $H$ and $\hat{H}$ contain compact open subgroups. Let $P$ be the periodic subgroup of $\hat{H}$; clearly $P$ is characteristic, open, and contains any compact open subgroup of $\hat{H}$. We may apply 2.1 to $P$ and obtain a compact open subgroup $K$ invariant under $B \hat{~}$. Now the annihilator $K^{\perp}=(\hat{G} / K)^{\wedge}=\mathbf{R}^{n} \times(\hat{H} / K)^{\wedge}=\mathbf{R}^{n} \times K_{1}$, where $K_{1}=(\hat{H} / K)^{\wedge}$ is a compact open subgroup of $H$ (compact because $\hat{H} / K$ is discrete and open because $\left(H / K_{1}\right)^{\wedge}=K$ is compact). Moreover $K^{\perp}$ is $B$ invariant because $K$ is $B^{\wedge}$ invariant. Since $K_{1}$ is maximal compact in $K^{\perp}=\mathrm{R}^{n} \times K_{1}, K_{1}$ is $B$ invariant as well. All together we have $\mathbf{R}^{n} \times K_{1}$ open in $G$ and a group $B$ of automorphisms leaving $\mathbf{R}^{n} \times K_{1}$ and $K_{1}$ invariant. It is easily checked that $\left(B \mid \mathbf{R}^{n} \times K_{1}\right)^{\wedge}$ is just the group of actions that $B^{\wedge}$ induces on $\hat{G} / K=\left(\mathbf{R}^{n} \times K_{1}\right)^{\wedge}$ and so $\left(\mathbf{R}^{n} \times K_{1}\right)^{\wedge}$ has precompact orbits under $\left(B \mid \mathbf{R}^{n} \times K_{1}\right)^{\wedge}$. We want to show $\mathbf{R}^{n} \times K_{1}$ has small $B$ invariant neighborhoods and so we are reduced to the following:

2.8 Lemma. Suppose $G=\mathbf{R}^{n} \times K$ is locally compact abelian and $\dot{\hat{G}}$ bas pre. compact $B^{\wedge}$ orbits, where $B$ is a group of automorphisms of $G$. Then $G$ bas small $B$ invariant neighborboods.

Proof. Let $P_{1}: G \rightarrow \mathbf{R}^{n}$ and $P_{2}: G \rightarrow K$ be the projections associated with the direct product. For $\alpha \in B$, let $\alpha_{1}=P_{1} \alpha P_{1}, \alpha_{2}=P_{2} \alpha P_{1}$, and $\alpha_{3}=P_{2} \alpha P_{2}$. Since $K$ is necessarily $B$ invariant $P_{1} \alpha P_{2}=0$ and so $a$ may be viewed as a matrix

$$
a=\left(\begin{array}{ll}
a_{1} & 0 \\
a_{2} & a_{3}
\end{array}\right) \text {. }
$$

Moreover if

$$
\beta=\left(\begin{array}{ll}
\beta_{1} & 0 \\
\beta_{2} & \beta_{3}
\end{array}\right)
$$

we have 


$$
\alpha \beta=\left(\begin{array}{cc}
\alpha_{1} \beta_{1} & 0 \\
\alpha_{2} \beta_{1}+\alpha_{3} \beta_{2} & \alpha_{3} \beta_{3}
\end{array}\right) .
$$

In particular, $P_{1} B P_{1}$ and $P_{2} B P_{2}$ are subgroups of $\mathscr{A}\left(\mathbf{R}^{n}\right)$ and $\mathfrak{A}(K)$ respectively.

For $(v, k) \in G$ we have $\alpha(v, k)=\left(\alpha_{1}(v), \alpha_{2}(v)+\alpha_{3}(k)\right)$. A direct calculation shows that, for $(\lambda, d) \in \hat{\mathbf{R}}^{n} \times \hat{K}=\hat{G}$, we have $\hat{\alpha}(\lambda, d)=\left(\hat{\alpha}_{1}(\lambda)+\hat{\alpha}_{2}(d), \hat{\alpha}_{3}(d)\right)$. Since $\{\hat{\alpha}(\lambda, d) \mid \alpha \in B\}^{-}$is compact for each fixed $(\lambda, d)$ we have $\left\{\hat{a}_{3}(d)\right\}$ finite for each fixed $d,\left\{\hat{\alpha}_{1}(\lambda)\right\}^{-}$compact for each fixed $\lambda$, and $\left\{\hat{\alpha}_{2}(d)\right\}^{-}$compact for each fixed $d$. It follows by 2.6 that $\mathbf{R}^{n}$ has small $P_{1} B P_{1}$ invariant neighborhoods and $K$ has small $P_{2} B P_{2}$ invariant neighborhoods. Since $\left\{\alpha_{2}(d)\right\}^{-}$is compact for each fixed $d$, so is $\bigcup_{a_{i} i}\left\{\hat{\alpha}_{2}\left(d_{i}\right)\right\}^{-}$for any finite set $d_{1}, \ldots, d_{n} \in \hat{K}$. By the Ascoli Theorem (p. 233 of [15]) the family $\bigcup_{a, i}\left\{\hat{\alpha}_{2}\left(d_{i}\right)\right\}$ is equicontinuous at 1 ; since sets of the form $W\left(d_{1}, \cdots, d_{n^{\prime}} \epsilon\right)$ form a base of neighborhoods of 1 in $K$ (see proof of 2.6), we may conclude that given any neighborhood $U_{1}$ of 1 in $K$ there is a neighborhood $U_{2}$ of 0 in $\mathbf{R}^{n}$ such that $\alpha_{2}\left(U_{2}\right) \subset U_{1}$ for every $a \in B$. It follows easily that given any neighborhood $U$ of 1 in $G$ there is a neighborhood $W$ of 1 in $G$ contained in $U$, with $\alpha(W) \subset U$ for every $\alpha \in B$. Then $\bigcup_{\alpha}(W)$ is a $B$ invariant neighborhood of 1 contained in $U$. Thus $G$ has small $B$ invariant neighborhoods and 2.8 and 2.7 are proved.

3. Representations of $[F C]^{-}$groups. In this section we show that a type I $[F C]^{-}$group has a Hausdorff dual space and consequently is CCR. We again assume $G$ is second countable.

3.1 Proposition. If $G \in[F C]^{-}$then $G \in[I N]$. If in addition $G$ is type $I$ then $G \in[F D]^{-}$and $G$ satisfies an exact sequence $(1) \rightarrow K \rightarrow G \rightarrow \mathbf{R}^{n} \times A \rightarrow(1)$, where $K$ is compact and $A$ is discrete abelian.

Proof. If $G \in[F C]^{-}$then $G \in[I N]$ by the Corollary to 2.2 , and moreover, by 2.2, $G$ satisfies an exact sequence $(1) \rightarrow K \rightarrow G \rightarrow \mathbf{R}^{n} \times D \rightarrow(1)$ where $K$ is compact and $D$ is discrete $F C$. If $G$ is type $I, D$ is type $I$, and therefore $D$ has a finite commutator by Theorem 5.4 of [11] and Theorem 5.3 of [23]. It follows that $G \in[F D]^{-}$and satisfies an exact sequence of the kind described.

3.2 Definition. The character $t \mapsto t$ of $T$ will be denoted $\chi_{0}$. If $G$ satisfies the central exact sequence $(1) \rightarrow T \rightarrow G \rightarrow H \rightarrow(1)$ then, for each $\chi \in \hat{T}$, we let

$$
\hat{G}_{\chi}=\{\pi \in \hat{G}|\pi| T=\chi \cdot I\}
$$

and

$$
G_{\chi}^{\mathrm{fac}}=\{\pi \mid \pi \text { a factor representation of } G \text { and } \pi \mid T=\chi \cdot I\} .
$$


We denote $\hat{G}_{\chi_{0}}$ by $\hat{G}_{1}$ and $G_{\chi_{0}}^{\mathrm{fac}}$ by $G_{1}^{\mathrm{fac}}$.

3.3 Lemma. Suppose $(1) \rightarrow T \rightarrow G \rightarrow H \rightarrow(1)$ is a central exact sequence of locally compact groups. Then every element of $G_{1}^{\mathrm{fac}}$ is type $\mathrm{I}$ if and only if $\hat{G}_{1}$ is a closed and open $T_{0}$ subspace of $\hat{G}$.

Proof. For each $\chi \in \hat{T}$ let $\frac{m_{\chi}}{\chi}$ be the central idempotent measure in $M^{1}(G)$ concentrated on $T$ and given by $\overline{\chi(t)} d t$ there, where $d t$ is normalized Haar measure on $T$. For each $f \in L^{1}(G)$ and $\pi \in \hat{G}$ we have $\left\|\pi\left(f * m_{\boldsymbol{\chi}}\right)\right\| \leq\left\|m_{\boldsymbol{\chi}}\right\|\|\pi(f)\|$; it follows that multiplication (convolution) by $m_{\boldsymbol{\chi}}$ is a continuous endomorphism of $C^{*}(G)$.

If $\pi \in G_{\chi}^{\mathrm{fac}}$, then $\pi\left(m_{\chi}\right)=I$ and $\pi\left(m_{\chi^{\prime}}\right)=0$ for $\chi \neq \chi^{\prime}$. Moreover it is easy to see that each $\hat{G}_{\chi}$ is open and closed in $\hat{G}$. It is then routine to verify the following: $C^{*}(G)=\bigoplus_{\hat{T}} C^{*}(G) m_{\chi}$, the $C^{*}$ direct sum of closed orthogonal two sided * ideals. In particular, we are asserting that for every $a \in C^{*}(G), a m_{\chi} \rightarrow 0$ as $\chi \rightarrow \pm \infty$. Furthermore if $\pi \in G_{a^{\prime}}^{\text {fac }}$, then $\operatorname{ker} \pi \supset \bigoplus_{\chi \neq \chi^{\prime}} C^{*}(G) m_{\chi}$. Thus to say that every element of $G_{1}^{\mathrm{fac}}$ is type $I$ is to assert that $C^{*}(G) m_{\chi_{0}}$ is type I; it follows that $G_{1}^{\mathrm{fac}}$ being type $\mathrm{I}$ is equivalent to $\hat{G}_{1}=\left(C^{*}(G) m_{\boldsymbol{\chi}_{0}}\right)^{\wedge}$ being $T_{0}$.

The proof of the following proposition is similar to a calculation in $\$ 6$, Chapter V, of [1].

3.4 Proposition. Consider the central extension of locally compact groups $(1) \rightarrow T \rightarrow G \rightarrow \mathbf{R}^{n} \oplus D \rightarrow(1)$, where $D$ is countable discrete abelian. If every element of $G_{1}^{\mathrm{fac}}$ is type I, then the map $\left.\pi \mapsto \pi\right|_{Z(G)}$ defines a bomeomorphism of $\hat{G}_{1}$ onto $\widehat{Z}_{(G)}$.

Proof. By Proposition 1.7 we may assume that the cocycle giving $G$ as the extension of $T$ by $\mathbf{R}^{n} \oplus D$ is chosen so that $\alpha(x, y)=1$ for all $x, y$ in the image of $Z(G)$ in $\mathbf{R}^{n} \oplus D$; that is, for all $x, y \in Z_{a}\left(\mathbf{R}^{n} \oplus D\right)$. Thus we may write $G=$ $T \times\left(\mathbf{R}^{n} \oplus D\right)$ with multiplication defined by $\langle s, x\rangle\langle t, y\rangle=\langle s t \alpha(x, y), x+y\rangle$ for all $s, t \in T$ and all $x, y \in \mathbf{R}^{n} \oplus D$ and the topology the unique locally compact group topology compatible with the Borel group structure and left invariant measure on $T \times\left(\mathbf{R}^{n} \oplus D\right)$. We then have (using the cocycle identity) that $\langle s, x\rangle\langle t, y\rangle\langle s, x\rangle^{-1}=\left\langle t \alpha(y, x) \alpha(y, x)^{-1}, y\right\rangle$ for all $s, t \in T^{-}$and $x, y \in \mathbf{R}^{n} \oplus D$.

Let $M$ be a maximal abelian subgroup of $G$. Then $M$ is closed and necessarily contains $T$. Therefore $M$ is normal. Furthermore since $T$ is injective in the category of locally compact abelian groups we may write $M=T \oplus A$ where $A$ is a closed abelian subgroup of $M$. The group $G$ acts by inner automorphisms on $M$, hence also on the character group $\hat{M}$. This action on $\hat{M}$ clearly preserves $\hat{M}_{1}=\left\{\chi_{0}\right\} \times \hat{A}$. We compute the action of $G$ on $\hat{M}_{1}:$ let $\left(\chi_{0}, \lambda\right) \in \hat{M}_{1}$, where $\lambda \in \hat{A}$, and let $\langle s, x\rangle \in G,\langle t, 0\rangle \in T$, and $\left\langle t^{\prime}, y\right\rangle \in A$. Then we have 


$$
\begin{aligned}
& \left(\chi_{0}, \lambda\right)^{\langle s, x\rangle}\left(\langle t, 0\rangle\left\langle t^{\prime}, y\right\rangle\right)=\left(\chi_{0}, \lambda\right)\left(\left\langle t t^{\prime} \alpha(x, y) \alpha(y, x)^{-1}, y\right\rangle\right) \\
& \quad=\left(\chi_{0}, \lambda\right)\left(\left\langle t \alpha(x, y) \alpha(y, x)^{-1}, 0\right\rangle\left\langle t^{\prime}, y\right\rangle\right)=t \alpha(x, y) \alpha(y, x)^{-1} \lambda\left(\left\langle t^{\prime}, y\right\rangle\right) .
\end{aligned}
$$

In other words, we have $\left(\chi_{0}, \lambda\right)^{\langle s, x\rangle}=\left(\chi_{0}, \lambda+\phi_{x}\right)$ where $\phi_{x} \in \hat{A}$ is defined by $\phi_{x}(\langle t, y\rangle)=\alpha(x, y) \alpha(y, x)^{-1}$ for all $\langle t, y\rangle \in A \circ \quad\left(\phi_{x}\right.$ is actually a continuous homomorphism by 1.7.) Moreover the map $\Phi: x \rightarrow \phi_{x}$ is a continuous homomorphism (see Proposition 1.1 of [16]) from $\mathbf{R}^{n} \oplus D$ to $\hat{A}$. Since $M$ is maximal abelian it is easy to see that the kernel of $\Phi$ is $\left\{x \in \mathbf{R}^{n} \oplus D \mid\langle 0, x\rangle \in M\right\}$. We may summarize our computation by saying that the orbit of $\left(\chi_{0}, \lambda\right)$ under $G$ is the set $\left\{\chi_{0}\right\} \times(\lambda+\operatorname{Range} \Phi)$, where $\lambda+\operatorname{Range} \Phi$ is the coset of the subgroup Range $\Phi$ in $\hat{A}$ which contains $\lambda$, and that the stable group of $\left(\chi_{0}, \lambda\right)$ is $M$.

We claim that if every element of $G_{1}^{\text {fac }}$ is type $I$, then Range $\Phi$ is closed. In fact, if Range $\Phi$ is not closed, find two distinct cosets of Range $\Phi$ contained in Range $\Phi$. These two cosets define two orbits in $\hat{M}_{1}$ contained in each other's closure. It follows by Theorem 4.3 of [7] that if $\pi_{1}$ and $\pi_{2}$ are characters chosen from the two orbits in $\hat{M}_{1}$, then the two induced representations $U^{\pi_{1}}$ and $U^{\pi_{2}}$ of $G$ weakly contain each other. Moreover by Theorems 7.6 and 8.1 of [19] these representations are irreducible and inequivalent. Thus $\hat{G}_{1}$ is not $T_{0}$ and by 3.3 there are elements of $G_{1}^{\mathrm{fac}}$ which are not type $I$. Thus if in fact every element of $G_{1}^{\text {fac }}$ is type $I$, then Range $\Phi$ is closed.

Since Range $\Phi$ is closed, the orbits in $\hat{M}_{1}$ under $G$ are all closed, and by Theorem 1 of [8] we get that every quasi-orbit concentrated in $\hat{M}_{1}$ is transitive. It follows by Theorems 7.6 and 8.1 of [19] that we have a bijection between $\hat{G}_{1}$ and the orbit space $\hat{M}_{1} / G$ as follows: given an element $\pi \in \hat{G}_{1},\left.\pi\right|_{M}=n \int_{\hat{M}_{1}} \sigma d \mu(\sigma)$ (where $n$ is an integer or $\infty$ ) for a unique measure class $\mu$ which is concentrated in an orbit $O(\mu)$ in $\hat{M}_{1}$; we associate the orbit $O(\mu)$ with $\pi$. Moreover given any element in $O(\mu)$ the induced representation (up to equivalence) is $\pi$. It is easy to see on the basis of Theorem 4.3 of [7] that this bijection is a homeomorphism, where $\hat{M}_{1} / G=\left\{\chi_{0}\right\} \times \hat{A} /$ Range $\Phi$ has the topology of $\hat{A} /$ Range $\Phi$.

Now let $r^{\prime}: \hat{A} \rightarrow(Z(G) \cap A)^{\wedge}$ be the restriction function. Then by 24.11 of [13], $r^{\prime}$ is continuous, open, surjective, and has kernel $(Z(G) \cap A)^{\perp}$, the annihilator of $Z(G) \cap A$ in $\hat{A}$. However it is easily seen that $Z(G) \cap A=\operatorname{ker} \Phi$, where $\Phi \stackrel{\wedge}{\wedge} \rightarrow\left(\mathbf{R}^{n} \oplus D\right)^{\wedge}$ is the adjoint map to $\Phi: \mathbf{R}^{n} \oplus D \rightarrow \hat{A}$. It follows by 24.38 of [13] that ker $r^{\prime}=$ Range $\Phi$. Thus $r^{\prime}$ induces a topological group isomorphism $\hat{A} /$ Range $\Phi \rightarrow(Z(G) \cap A)^{\hat{\imath}}$.

Since $M=T \oplus A$ and $T \subset Z(G) \subset M$, we have $Z(G)=T \oplus(Z(G) \cap A)$. It follows that $Z(G)_{1}=\left\{\chi_{0}\right\} \times(Z(G) \cap A)$; consequently the restriction $r: \hat{M}_{1} \rightarrow$ $Z(G)_{1}$ induces a homeomorphism $s$ of $\hat{M}_{1} / G$ onto $Z(G)_{1}$. It is clear that, for $\left.\pi \in \hat{G}_{1} \pi\right|_{Z(G)}=s(O(\mu)) \cdot I$, and the proposition is proved. 
Remark. Let $a \in H^{2}\left(\mathbf{R}^{n} \oplus D, T\right)$ and let $a$ be a cocycle representing $a$ such that $a(x, y)=1$ for all $x, y \in Z_{\alpha}\left(\mathbf{R}^{n} \oplus D\right)$. Let $H$ be the group satisfying the central exact sequence $(1) \rightarrow T \rightarrow H \rightarrow \mathbf{R}^{n} \oplus D \rightarrow(1)$ and defined by $\alpha$ in the usual way. Let $\sigma: \mathbf{R}^{n} \oplus D \rightarrow H$ be a Borel section and $\pi \mapsto \pi \circ \sigma$ the bijection

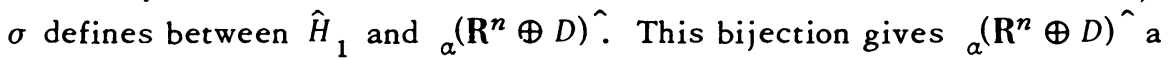
topology which is independent of the choice of $\sigma$ since $\pi \circ \sigma$ and $\pi \circ \sigma^{\prime}$ differ only by tensoring with an element of $\left(R^{n} \oplus D\right)^{\wedge}$ for different Borel sections $\sigma$ and $\sigma^{\prime}$ (see pp. 25-26 of [1]).

Corollary to 3.4. Let $a \in H^{2}\left(\mathbf{R}^{n} \oplus D, T\right)$ and let a be a cocycle representing a sucb that a is trivial on $Z_{a}\left(\mathbf{R}^{n} \oplus D\right)$. If every factor a representation of $\mathbf{R}^{n} \oplus D$ is type I, then $\pi \mapsto \pi \mid Z_{a}\left(\mathbf{R}^{n} \oplus D\right)$ defines a bomeomorphism of ${ }_{a}\left(\mathbf{R}^{n} \oplus D\right)^{\wedge}$ onto $Z_{a}\left(\mathbf{R}^{n} \oplus D\right)$.

Proof. Let $H$ be the group defined by $\alpha$, so that (1) $\rightarrow T \rightarrow H \rightarrow \mathbf{R}^{n} \oplus D$ $\rightarrow(1)$ is central exact. Then $H_{1}^{\text {fac }}$ consists of type I elements, and so by 3.4 the map $\pi \mapsto \pi \mid Z(H)$ defines a homeomorphism from $\hat{H}_{1}$ onto $Z(H)$. To prove the corollary, we need only show that for the given Borel section $\sigma: \mathbf{R}^{n} \oplus D \rightarrow H$ (i.e., $\sigma(x)=\langle 0, x\rangle$ ), then $\chi \mapsto \chi \circ \sigma$ is a homeomorphism from $Z(H)_{1}$ onto $Z_{\alpha}\left(\mathbf{R}^{n} \oplus D\right)^{\wedge}$. Since $\alpha$ is trivial on $Z_{\alpha}\left(\mathbf{R}^{n} \oplus D\right), \sigma \mid Z_{a}\left(\mathbf{R}^{n} \oplus D\right)$ is a group isomorphism and hence continuous. (In fact, any Borel homomorphism from a second countable locally compact group into a second countable locally compact group is continuous - see pp. 14-15 of [1].) Reasoning as in 1.7 we may find a section $\sigma^{\prime}$ which gives rise to $\alpha$ and which is a topological group isomorphism when restricted to $Z_{\alpha}\left(\mathbf{R}^{n} \oplus D\right)$. Since $\sigma$ and $\sigma^{\prime}$ differ by an element of $\left(\mathbf{R}^{n} \oplus D\right)^{\hat{2}}$ we conclude that $\sigma \mid Z_{\alpha}\left(\mathbf{R}^{n} \oplus D\right)$ is a topological group isomorphism, so that $\sigma\left(Z_{a}\left(\mathbf{R}^{n} \oplus D\right)\right)$ is a closed complementary subgroup to $T$ in $Z(H)$ and $\chi \mapsto$ $\chi \circ \sigma$ provides a homeomorphism from $\widehat{Z(H)}{ }_{1}$ onto $Z_{\alpha}\left(\mathbf{R}^{n} \oplus D\right)^{\wedge}$

3.5 Proposition. Let $G$ satisfy the central exact sequence (1) $\rightarrow T \rightarrow G$ $\rightarrow \mathbf{R}^{n} \rightarrow(1)$. Then every element of $G_{1}^{\mathrm{fac}}$ is type I and therefore $\pi \mapsto \pi \mid Z(G)$ is a bomeomorphism from $\hat{G}_{1}$ onto $\widehat{Z(G)} \widehat{1}_{1}$.

Remark. This is of course a very special case of Kirillov's results in Unitary representations of nilpotent Lie groups (Uspehi Mat. Nauk 17 (1962), 57-110). However the proof we give is short and in the spirit of 3.4 , so we include it for completeness.

Proof. The following diagram is exact and commutative.

$$
\begin{aligned}
& (0) \rightarrow L(T) \rightarrow L(G) \rightarrow L\left(\mathbf{R}^{n}\right) \rightarrow(0) \\
& (1) \rightarrow \frac{\downarrow}{T} \rightarrow \stackrel{\downarrow}{G} \rightarrow \stackrel{\downarrow}{\mathbf{R}^{n}} \rightarrow \text { (1) }
\end{aligned}
$$


Here the top line consists of Lie algebras and the vertical maps are exponential maps. Let $\sigma^{\prime}$ be a linear section from $L\left(\mathbf{R}^{n}\right)$ to $L(G)$, and let $\sigma=\exp _{G} \circ \sigma^{\prime}$ 。 $\left(\exp _{\mathbf{R}^{n}}\right)^{-1}$. Then $\sigma: \mathbf{R}^{n} \rightarrow G$ is a section and the associated cocycle is defined by $\alpha(x, y)=\sigma(x) \sigma(y) \sigma(x+y)^{-1}$ for all $x, y \in \mathbf{R}^{n}$; a simple application of the Campbell-Hausdorff formula yields $a(x, y)=\exp _{G}\left(1 / 2\left[\sigma^{\prime}(X), \sigma^{\prime}(Y)\right]\right)$ where $\exp _{\mathbf{R}^{n}}(X)=x$ and $\exp _{\mathrm{R}^{n}}(Y)=y$. In other words, $\alpha(x, y)=e^{i B(x, y)}$ where $B$ is an alternating bilinear form on $\mathbf{R}^{n}$. Since $\sigma$ is continuous, $G$ is homeomorphic to $T \times \mathbf{R}^{n}$ and a gives the multiplication of $G$ in the usual way $(\langle s, x\rangle\langle t, y\rangle=$ $\langle\operatorname{sta}(x, y), x+y\rangle)$.

Now take $A$ to be a maximal subspace of $\mathbf{R}^{n}$ such that $B(x, y)=0$ for all $x, y \in A$. It is easily seen that the preimage of $A$ in $G$ is a maximal abelian subgroup $M$. Moreover, $\left.\sigma\right|_{A}$ is a topological group isomorphism and $\sigma(A)$ is a closed vector subgroup of $M$. We have that $M=T \oplus \sigma(A)$; let us regard $\sigma(A)$ as $A$ and $M=T \oplus A$. If we apply the arguments from the preceding proposition to $G$, we may take the $\alpha, M$, and $A$ obtained in this proposition to be the corresponding entities used in the proof of the last proposition. In particular, we now have that $\Phi: \mathbf{R}^{n} \rightarrow \hat{A}$, where $\hat{A}$ is a vector group and $\Phi$ is linear with its range automatically closed. Hence the arguments used in 3.4 go through for $G$, and we obtain that $\hat{G}_{1}$ is $T_{2}$ and by $3.3 G_{1}^{\text {fac }}$ is type $I$. This proves 3.5 .

3.6 Theorem. Suppose that $G \in[F C]^{-}$is type I. Then $\hat{G}$ is Hausdorff and $G$ is $C C R$.

Proof. By 3.1, $G$ satisfies an exact sequence (1) $\rightarrow K \rightarrow G \rightarrow \mathbf{R}^{n} \times A \rightarrow(1)$ where $K$ is compact and $A$ is compact and $A$ is countable discrete abelian. Since $\hat{K}$ is countable discrete we certainly have that $K$ is regularly embedded in $G$; that is, every element of $\hat{G}$ is obtained from $\hat{K}$ via the Mackey procedure. For each $\sigma \in \hat{K}$ let $\hat{G}_{\sigma}$ be the set of $\pi \in \hat{G}$ whose restrictions to $K$ all include $\sigma$ as a subrepresentation. We then have $\hat{G}_{\sigma}=\hat{G}_{\tau}$ if $\sigma$ is in the orbit of $\tau$ under $G$ and $\hat{G}_{\sigma}$ and $\hat{G}_{\tau}$ are disjoint if $\sigma$ is not in the orbit of $\tau$. Using the \# operation of $K$ and the orthogonality relations for characters of $K$, one may see easily that each $\hat{G}_{\sigma}$ is open and closed in $\hat{G}$. Therefore we will confine our attention to a single $\hat{G}_{\sigma}$.

Let $H(\sigma)$ be the stability group of $\sigma$ in $G$. Let $\widehat{H(\sigma)_{\sigma}}$ be the set of all elements of $\overbrace{H(\sigma)}$ whose restrictions to $K$ include (and hence are multiples of) $\sigma$, and let $H(\sigma)_{\sigma}^{\mathrm{fac}}$ be the set of all factor representations of $H(\sigma)$ whose restrictions to $K$ are multiples of $\sigma$. We see as above that $\widehat{H}_{(\sigma)_{\sigma}}$ is open and closed in $\widehat{H(\sigma)}$. Now $H(\sigma) / K$ is a closed subgroup of $\mathbf{R}^{n} \oplus A$ and is therefore of the form $\mathbf{R}^{m} \oplus D^{\prime}$ where $D^{\prime}$ is countable discrete abelian. Therefore by Theorem 8.2 
of [19] (see also the discussion on p. 60 of [1]) and by 1.7 there exists a cocycle $\alpha$ of $\mathbf{R}^{m} \oplus D^{\prime}$ such that $\alpha$ is trivial on $Z_{\alpha}\left(\mathbf{R}^{m} \oplus D^{\prime}\right)$ and an $\bar{\alpha}$ representation $\sigma^{\prime}$ of $H(\sigma)$ which extends $\sigma$. Here $\bar{a}$ denotes the inflacion of a to $H(\sigma)$. Moreover, by Theorem 8.3 of [19] the map $\lambda \rightarrow \bar{\lambda} \otimes \sigma^{\prime}$ is a bijection between equivalence classes of irreducible (resp. factor) $\alpha^{-1}$ representations of $H(\sigma) / K$ and $\widehat{H(\sigma)_{\sigma}}$ (resp. $H(\sigma)_{\sigma}^{\mathrm{fac}}$ ), where $\bar{\lambda}$ denotes the lifting of $\lambda$ to $H(\sigma)$. Since $G$ is

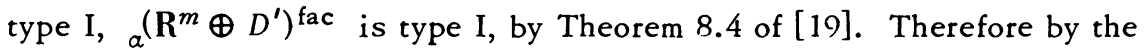

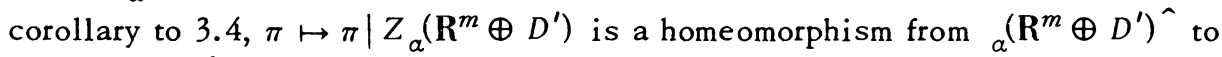
$Z_{a}\left(\mathbf{R}^{m} \oplus D^{\prime}\right)^{\wedge}$. We will use this fact to show that the bijection between ${ }_{a}\left(\mathbf{R}^{m} \oplus D^{\prime}\right)^{\wedge}$ and $\widehat{H(\sigma)_{\sigma}}$ is a homeomorphism and that consequently $\widehat{H(\sigma)}_{\sigma}$ is Hausdorff. It will follow easily that $\hat{G}_{\sigma}$ is Hausdorff.

To see that the bijection between $\left(\mathbf{R}^{m} \oplus D^{\prime}\right)^{\wedge}$ and ${ }_{H(\sigma)_{\sigma}}$ is a homeomor-

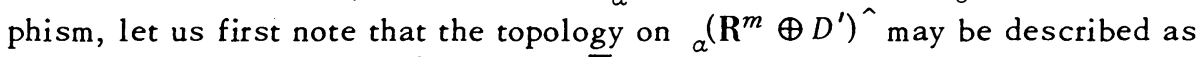
follows: If $S \subset a_{a}\left(\mathbf{R}^{m} \oplus D^{\prime}\right)^{\wedge}$, then $\lambda \in \bar{S}$ if and only if there are elements $\lambda_{n} \epsilon$ $\alpha^{\left(\mathbf{R}^{m} \oplus D^{\prime}\right)}$ and vectors $v_{n}$ and $v$ such that the functions $\left(\lambda_{n} v_{n}, v_{n}\right) \rightarrow(\lambda v, v)$ uniformly on $\mathbf{R}^{m} \oplus D^{\prime}$ compacta. This follows easily from the analogous description of the topology in $\hat{H}_{1}$ (where $(1) \rightarrow T \rightarrow H \rightarrow \mathbf{R}^{m} \oplus D^{\prime} \rightarrow(1)$ is the extension defined by $\alpha$ ) and the fact that a set in $\mathbf{R}^{m} \oplus D^{\prime}$ is compact if and only if its preimage in $H$ is compact. Now let $S \subset_{a}\left(\mathbf{R}^{m} \oplus D^{\prime}\right)^{\wedge}$ and suppose that the image of $S$ in $\widehat{H(\sigma)}$ is closed. If $\lambda \in \bar{S}$ there are elements $\lambda_{n}$ in $S$ with vectors $v_{n}$ such that the functions $\left(\lambda_{n} v_{n}, v_{n}\right) \rightarrow(\lambda v, v)$ uniformly on $\mathbf{R}^{m} \oplus D^{\prime}$ compacta for some vector $v$. Choose any vector $w$ in the representation space of $\sigma^{\prime}$. Then $\left(\bar{\lambda}_{n} \otimes \sigma^{\prime}\left(v_{n} \otimes w\right), v_{n} \otimes w\right) \rightarrow\left(\bar{\lambda} \otimes \sigma^{\prime}(v \otimes w), v \otimes w\right)$ uniformly on $H(\sigma)$ compacta. Hence $\bar{\lambda} \otimes \sigma^{\prime}$ is in the image of $S$ and $\lambda \in S$. Thus $S$ is closed and $\lambda \mapsto \bar{\lambda} \otimes \sigma^{\prime}$ is continuous.

Now suppose that $S \subset{ }_{a}\left(\mathbf{R}^{m} \oplus D^{\prime}\right)^{\wedge}$ is closed and that $\bar{\lambda} \otimes \sigma^{\prime}$ is in the closure of the image of $S$ in $\widehat{H(\sigma)}_{\sigma}$. Then there are elements $\lambda_{n} \in S$ and vectors $v_{n}$ and $v$ such that the functions $\left(\bar{\lambda}_{n} \otimes \sigma^{\prime} v_{n}, v_{n}\right) \rightarrow\left(\bar{\lambda} \otimes \sigma^{\prime} v, v\right)$ uniformly on

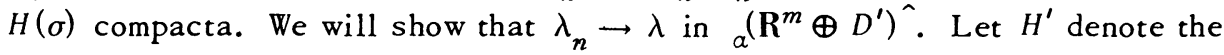
preimage of $Z_{\alpha}\left(\mathbf{R}^{m} \oplus D^{\prime}\right)$ in $H(\sigma)$, so that $H^{\prime} / K=Z_{\alpha}\left(\mathbf{R}^{m} \oplus D^{\prime}\right)$. Since $\lambda_{n}$ and $\lambda$ are irreducible $\alpha^{-1}$-representations and $\alpha \mid Z_{\alpha}\left(\mathbf{R}^{m} \oplus D^{\prime}\right)$ is trivial, we have by Schur's lemma that $\lambda_{n} \mid 7 \cdot\left(\mathbf{R}^{m} \oplus D^{\prime}\right)=\chi_{n} \cdot I$ and $\lambda \mid Z_{\alpha}\left(\mathbf{R}^{m} \oplus D^{\prime}\right)=\chi \cdot I$ for characters $\chi_{n}$ and $\chi$ of the abelian group $Z_{a}\left(\mathbf{R}^{m} \oplus D^{\prime}\right)$. Denote by $\bar{\chi}_{n}$ and $\bar{\chi}$ the liftings of these characters to $H^{\prime}$. We then have

$$
\begin{aligned}
\left(\left(\lambda_{n} \otimes \sigma^{\prime}\right) \mid H^{\prime} v_{n^{\prime}} v_{n}\right) & =\bar{\chi}_{n} \cdot\left(m_{n} \sigma^{\prime} \mid H^{\prime} v_{n^{\prime}}, v_{n}\right) \rightarrow \bar{\chi} \cdot\left(m \sigma^{\prime} \mid H^{\prime} v, v\right) \\
& =\left(\left(\bar{\lambda} \otimes \sigma^{\prime}\right) \mid H^{\prime} v, v\right)
\end{aligned}
$$


uniformly on $H^{\prime}$ compacta where $m_{n}$ and $m$ are positive integers or $+\infty$. Moreover, $\sigma^{\prime} \mid H^{\prime}$ is an ordinary finite dimensional representation of $H^{\prime}$, since $\sigma^{\prime}$ is an $\bar{\alpha}$-representation of $H(\sigma)$ and $\bar{\alpha}$ is trivial on $H^{\prime}$. We will show that $\bar{\chi}_{n} \rightarrow \bar{\chi}$ uniformly on $H^{\prime}$ compacta. To do this, divide $H^{\prime}$ by the intersection of the kernels of $\left\{\chi_{n} \sigma^{\prime}\right\}$ and $\chi^{\sigma^{\prime}}$, and obtain thereby an $[F D]^{-} \cap[M A P]$ group $H^{\circ}$. It is easy to see (on the basis of 17.3.7 of [5]) that such a group must be [SIN]; consequently it has a \# operation by 1.3. Now $\bar{\chi}_{n}\left(m_{n} \sigma^{\prime} \mid H^{\prime} v_{n}, v_{n}\right) \rightarrow \bar{\chi}^{\left(m \sigma^{\prime} \mid H^{\prime} v, v\right)}$ uniformly on $H^{\circ}$ compacta, and using 1.6 we have $\left(k \sigma^{\prime} \mid H^{\prime}\right)^{\#}=\left(\operatorname{deg} \sigma^{\prime} \mid H^{\prime}\right)^{-1}$ - $\operatorname{Tr}\left(\sigma^{\prime} \mid H^{\prime}\right)$ for $k$ an integer or $\infty$. Since $\bar{\chi}_{n}$ and $\bar{\chi}$ are central we have $\bar{\chi}_{n} \operatorname{Tr}\left(\sigma^{\prime} \mid H^{\prime}\right) \rightarrow \bar{\chi} \operatorname{Tr}\left(\sigma^{\prime} \mid H^{\prime}\right)$ uniformly on $H^{\circ}$ compacta, hence also on $H^{\prime}$ compacta. Since $\sigma^{\prime} \mid K=\sigma$ is irreducible and finite dimensional, the linear span of $\left\{\sigma_{k} \mid k \in K\right\}$ is a full matrix algebra. Therefore any $K$ coset in $H^{\prime}$ contains an $x$ with $\operatorname{Tr} \sigma_{x}^{\prime} \neq 0$. It follows by the continuity of $\sigma^{\prime}$ that there is a neighborhood $U$ of $x$ with $\left|\operatorname{Tr} \sigma_{y}^{\prime}\right| \geq \epsilon>0$ for all $y \in U$. Certainly $\bar{\chi}_{n} \rightarrow \bar{\chi}$ uniformly on $U$, and since $\bar{\chi}_{n}$ and $\bar{\chi}$ are constant on $K$ cosets, a simple compactness argument yields convergence uniformly on $H^{\prime}$ compacta. Therefore $\lambda_{n} \mid Z_{a}\left(\mathbf{R}^{m} \oplus D^{\prime}\right)=\chi_{n}$. $I \rightarrow \chi \cdot I=\lambda \mid Z_{\alpha}\left(\mathbf{R}^{m} \oplus D^{\prime}\right)$ uniformly on $\mathrm{Z}_{a}\left(\mathbf{R}^{m} \oplus D^{\prime}\right)$ compacta; it follows by the

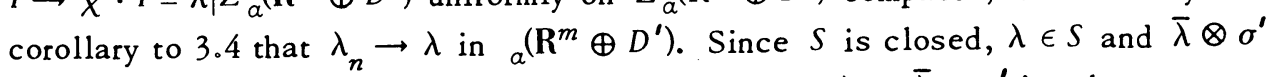
is in the image of $S$. Thus this image is closed, and $\lambda \rightarrow \bar{\lambda} \otimes \sigma^{\prime}$ is a homeomorphism. It follows that $\overbrace{H(\sigma)}$ is Hausdorff, since $a^{\left(\mathbf{R}^{m} \oplus D^{\prime}\right)^{\wedge}}$ is Hausdorff. By Theorem 8.1 of [19], inducing gives a bijection between $\hat{H(\sigma)}_{\sigma}$ and $\hat{G}_{\sigma}$. By Theorem 4.3 of [7], this bijection is a homeomorphism. (To see that this map is open, one must also use the orthogonality relations for $K_{\text {. }}$ ) Since $\hat{G}$ is the disjoint union of the open and closed sets $\hat{G}_{\sigma}, \hat{G}$ is Hausdorff. This proves 3.6.

3.7 Theorem. Suppose $G$ is almost connected $[F C]^{-}$. Then $G$ satisfies an exact sequence $(1) \rightarrow K \rightarrow G \rightarrow \mathbf{R}^{n} \rightarrow(1)$ for some compact normal subgroup $K$. Moreover $G$ is type $\mathrm{I}$ and the preceding theorem applies.

Proof. Since $G$ is almost connected, the group $D$ in 2.2 must be finite and so by enlarging $K$ we may assume $D$ is trivial. Since $\hat{K}$ is discrete, $K$ is regularly embedded in $G$ and the Mackey procedure yields all of $\hat{G}$. Take $\pi \in \hat{K}$. Then since $\mathbf{R}^{n} \times \hat{K} \rightarrow \hat{K}$ is jointly continuous and $\mathbf{R}^{n}$ is connected, the stable group $H(\pi)=G$. Thus every element of $G^{\text {fac }}$ whose restriction to $K$ includes $\pi$ is of the form $\lambda \otimes \pi^{\prime}$, where $\pi^{\prime}$ is an $\mathbf{R}^{n}$ cocycle representation of $G$ extending $\pi$ and where $\lambda$ is a cocycle factor representation of $\mathbf{R}^{n}$ lifted to $G$. By 3.5, $\lambda$ must be type I. Thus $\lambda \otimes \pi^{\prime}$ is type I, and we have that every element of $G^{\text {fac }}$ is type I. Thus $G$ is type $I$.

4. Representations of type I [IN] groups. In this section we show on the basis of the preceding section that type $\mathrm{I}[I N]$ groups are CCR. The proof of 
4.1 below follows arguments of Thoma in [27] and uses the succeeding three lemmas.

4.1 Proposition. If $G \in[I N]$ is type $\mathrm{I}$, then $\left[G: G_{F}\right]<\infty$, where $G_{F}$ is the subgroup of all elements with precompact conjugacy classes.

4.2 Lemma. Let $f$ be the characteristic function of a compact invariant neighborbood of 1 in $G$, and let $\psi=f * f^{\sim}$. Then $\psi$ is continuous positive definite and vanishes at $\infty$. If $k^{2} \psi \geq \phi$ is a positive definite function for some real $k$ and some continuous positive definite $\phi$ (i.e., $k^{2} \psi=\phi+\gamma$ where $\gamma$ is also positive definite) then $\phi$ tends to 0 at $\infty$. If $\phi$ is central and continuous, then supp $\phi \subset G_{F}$.

Proof. According to 13.4 .11 of [5], $\psi$ is positive definite and continuous and in fact $\psi(x)=(\lambda(x) f, f)$, where $\lambda$ is the left regular representation of $G$ and $f \in L^{2}(G)$. Let $H$ be the closed cyclic subspace of $L^{2}(G)$ generated by $\{\lambda(x) f\}$. Then since $k^{2} \psi(x)=(\lambda(x) k f, k f)$, it follows by I, p. 398, of [22] that $\phi(x)=$ $(B \lambda(x) f, f)$ where $B: H \rightarrow H$ is a positive selfadjoint operator which commutes with every $\lambda(x) \mid H$. Now $A=B^{1 / 2}$ is a positive operator in the norm closed algebra generated by $B$, and it follows that $\phi(x)=(\lambda(x) k A f, k A f)$. Since $k A f \epsilon$ $H \subset L^{2}(G)$, it follows from 13.4 .11 of [5] that $\phi$ tends to zero at $\infty$. Now suppose $\phi$ is central. Since $G \in[I N], G_{F}$ is open. Since $\phi$ is constant on conjugacy classes and vanishes at $\infty$, it cannot assume a nonzero value at any element of $G$ whose conjugacy class is not precompact. Thus supp $\phi \subset G_{F}$.

The following lemma is a special case of Theorem 1 of [3].

4.3 Lemma. Let $N$ be an open normal subgroup of $G$ and $\phi$ a positive definite function vanishing off $N$. Suppose that $\pi_{\phi}$ is the cyclic representation of $G$ corresponding to $\phi$ and $\pi_{\phi \mid N}$ is the cyclic representation of $N$ corresponding to $\phi \mid N$. Then the induced representation $U^{\pi_{\phi} \mid N} \cong \pi_{\phi}$.

4.4 Lemma. We adopt the notation of 1.1. Suppose $G \in[I N]$. Then $\Phi_{c}$ contains a nonzero extreme point $\phi$ which is approacbed weak ${ }^{*}$ (in $L^{\infty}(G)=L^{1}(G)^{\prime}$ ) by elements of $\Phi_{c}$ which vanish off $G_{F}$.

Proof. First let us define the function $\psi$ as follows: let $f$ be the characteristic function of a compact invariant neighborhood of 1 and $f^{\sim}$ the usual adjoint $\left(f^{\sim}(x)=\overline{\left.f\left(x^{-1}\right)\right)}\right.$. Now let $\alpha=f * f^{\sim}$. Then $\alpha$ is continuous of compact support and positive definite. Since $f$ and $f^{\sim} \in Z\left(L^{1}(G)\right), a \in Z\left(L^{1}(G)\right)$ and so $\alpha$ is central. (See for example p. 157 of Loomis, Abstract barmonic analysis.) We take $\psi$ to be $a$ normalized at 1 .

The dual space $L^{\infty}(G)$ of $L^{1}(G)$ is locally convex in the weak ${ }^{*}$ topology. Moreover, as noted in $1.1, \phi$ is compact metric in this topology. It is convex as well, and so by the metrizable version of Choquet's Theorem (pp. $19 \mathrm{ff}$, of [24]) 
we have a regular positive Borel measure $\nu$ of total mass 1 such that $F(\psi)=$ $\int_{\Phi_{C}} F(\beta) d \nu(\beta)$ for every weak ${ }^{*}$ continuous linear functional $F$. Moreover the extreme points $K$ of $\Phi_{c}$ form a Borel set and $\nu\left(\Phi_{c} \backslash K\right)=0$.

Let us suppose that $\phi$ is in the topological support of $\nu$; i.e., that $\phi$ has no neighborhood $U$ with $\nu(U)=0$. Given weak ${ }^{*}$ continuous linear functionals $F_{1}, \ldots, F_{n}$ and $\epsilon>0$, define the neighborhood $U$ of $\phi$ by $U=\left\{\beta \in \Phi|| F_{i}(\beta)-\right.$ $F_{i}(\phi) \mid<\epsilon$ for $\left.i=1, \ldots, n\right\}$. Then since $\phi$ is in the topological support of $\nu$ there is a continuous positive function $g$ on $\Phi_{c}$ with supp $g \subset U$ and $\int g(\beta) d \nu(\beta)=1$. Let $\beta_{g}=\int g(\beta) \beta d \nu(\beta)$. We mean by this that $\beta_{g}$ is the unique continuous central positive definite function in $\Phi_{c}$ with $F\left(\beta_{g}\right)=$ $\int g(\beta) F(\beta) d \nu(\beta)$ for every continuous linear functional $F$ (see Proposition 1.1 of [24]). Now for $i=1,2, \ldots, n$ we have

$$
\begin{aligned}
\left|F_{i}\left(\beta_{g}\right)-F_{i}(\phi)\right| & =\left|\int g(\beta)\left(F_{i}(\beta)-F_{i}(\phi)\right) d \nu(\beta)\right| \\
& \leq \max _{\beta \in U}\left|F_{i}(\beta)-F_{i}(\phi)\right| \int g(\beta) d \nu(\beta) \leq \epsilon .
\end{aligned}
$$

Thus given $F_{1}, \cdots, F_{n}$ and $\epsilon>0$ there is a $\beta_{g} \epsilon \Phi_{c}$ with $\left|F_{i}\left(\beta_{g}\right)-F_{i}(\phi)\right|<\epsilon$ for each $i$. If $\lambda=\max g$ then $\lambda \psi \geq \beta_{g}$ as positive definite functions (in fact, if $\langle$,$\rangle denotes the pairing between L^{1}$ and $L^{\infty}$, then for $f \in L^{1},\left\langle f^{\sim} * f, \beta_{g}\right\rangle=$ $\left.\left.\int g(\beta)\left\langle f^{\nu} * f, \beta\right\rangle d \nu(\beta) \leq \lambda \int \zeta^{\sim} * f, \beta\right\rangle d \nu(\beta)=\left\langle f^{\nu} * f, \lambda \psi\right\rangle\right)$. Therefore $\beta_{g}$ vanishes off $G_{F}$ by 4.2 .

To complete the proof we note that $\phi$ may be taken to be a nonzero extreme point of $\Phi_{c}$. Suppose that (top supp $\left.\nu\right) \cap K \subset\{0\}$. Then since $\Phi_{c} \backslash\{0\}=$ $\left(\Phi_{c} \backslash K\right) \cup(K \backslash\{0\})$, we have $\nu\left(\Phi_{c} \backslash\{0\}\right)=0$ and $\psi=0$, a contradiction. Therefore the topological support of $\nu$ includes nonzero extreme points, and these are approached weak ${ }^{*}$ by elements $\beta_{g}$ of $\Phi_{c}$ which vanish off $G_{F}$.

Proof of 4.1. Since $G \in[i N], G_{F}$ is open in $G$ and it is certainly normal. By Proposition 17.3.5 of [5] the extreme points of $\Phi_{c}$ are 0 and the characters of finite type taking the value 1 at 1 . Characters of finite type are associated as positive definite functions with cyclic factor representations of type $\mathrm{I}_{n}$ or type II $_{1}$. Since $G$ is type I these factor representations generate finite dimensional von Neumann algebras and since they are cyclic they must be finite dimensional representations of $G$ by 15.5 .3 of [5].

By 4.4 there is a character $\phi$ of finite type which is approached weak ${ }^{*}$ by elements of $\Phi_{c}$ which vanish off $G_{F}$. We see easily that $\int_{G} f(x) \phi(x) d x=0$ for all $f \in L^{1}(G)$ which vanish on $G_{F}$, and hence $\phi$ vanishes off $G_{F}$. It follows from 4.3 that the representation $\pi_{\phi}$ corresponding to $\phi$ is induced to $G$ from $G_{F}$ and since $\pi_{\phi}$ is finite dimensional we have $\left[G: G_{F}\right]<\infty$. 
The following was pointed out to the author by J. M. G. Fell.

4.5 Proposition. An open subgroup $H$ of a type I group $G$ is type I.

Proof. One may verify easily that $C^{*}(H)$ is a $C^{*}$ subalgebra of $C^{*}(G)$. Then the proposition follows from 4.3 .5 of [5].

4.6 Theorem. A type I [IN] group is CCR. An almost connected [IN] group is type I and bence CCR.

Proof. If $G$ is type I $[I N], G_{F}$ is type $I[F C]^{-}$by 4.5 and therefore CCR by 3.6. If on the other hand $G$ is almost connected [IN], then $G_{F}$ is almost connected $[F C]^{-}$and so $C C R$ by 3.7. Using 4.1 and the fact that an almost connected group can have no open subgroup of infinite index, we have that $\left[G: G_{F}\right]<\infty$ in either case. Since $G_{F}$ is CCR, it follows that $G$ is also CCR. (In fact Schochetman in 4.3 of [26] shows that a compact extension of a CCR group is CCR.)

As a corollary, we have the following widely known result. This was obtained also by E. Kaniuth [14], who showed that an [SIN] group with type I regular representation has only finite dimensional irreducible representations, and in the $[F C]^{-} \cap[S I N]$ case also by R. Mosak [21].

Corollary to 4.6. A type I [SIN] group has only finite dimensional irreducible representations.

Proof. In $G \in[S I N]$ then $L^{1}(G)$ has a central approximate identity and so every irreducible image of $C^{*}(G)$ has nonzero central elements. Since these images are algebras of all compact operators on various Hilbert spaces, the spaces in question must be finite dimensional.

5. [IN] groups with Hausdorff duals. We have already shown that a type I $[F C]^{-}$group has Hausdorff dual. We show in this section that an [IN] group with Hausdorff dual is $[F C]^{-}$and that an [SIN] group with Hausdorff dual is the projective limit of $[Z]$ groups.

5.1 Lemma. If $G$ is a noncompact locally compact abelian group and $H_{1}, \cdots, H_{n}$ are noncompact closed subgroups, there is a sequence $\left\{\chi_{m}\right\} \subset \hat{G}$ such that $\chi_{m} \mid H_{j}$ is nontrivial for each $m$ and $j$, and $\chi_{m} \rightarrow 1$ uniformly on compacta.

Proof. We dualize. We have $\hat{G}$ is nondiscrete locally compact abelian group, and $\left(G / H_{i}\right)^{\wedge}$ a closed subgroup for each $i$. Also, $\hat{H}_{i}=\hat{G} /\left(G / H_{i}\right)^{\wedge}$ is not discrete and so $\left(G / H_{i}\right)^{\wedge}$ is nowhere dense in $\hat{G}$ for each $i$. Now if the lemma were false there would be an open neighbrohood $N$ of 1 in $\hat{G}$ such that 
$N \subset \bigcup_{i=1}^{n}\left(G / H_{i}\right)^{\wedge}$. We have $N$ locally compact in the relative topology and $\left(G / H_{i}\right)^{-} \cap N$ nowhere dense in the relative topology. This contradicts the Baire category theorem, and the lemma is proved.

5.2 Theorem. Suppose $G \in[I N]$. Then $\hat{G}$ is Hausdorff if and only if $G \in$ $[F C]$ and $G$ is type I.

Proof. The "if" part is 3.6. Now suppose $G \in[I N]$ and $\hat{G}$ is $T_{2}$. Since $G$ is type $I,\left[G: G_{F}\right]<\infty$ by 4.1 . By 3.1 and $4.5, G_{F} \in[F D]^{-}$. Let $K$ be the closure of the commutator subgroup of $G_{F}$, and let $x_{1}, \cdots, x_{n}$ be coset representatives for $G_{F}$ in $G$ excluding a representative for the coset $G_{F}$ itself. We are assuming here that $G \neq G_{F}$ and will derive a contradiction. For $i=1,2, \cdots, n$, let $F_{i}$ be the closed subgroup of $G_{F}$ generated by the set $\left\{x_{i} b x_{i}^{-1} b^{-1} \mid b \in G_{F}\right\}$. Since $x_{i} \notin G_{F}, x_{i}^{-1}$ does not have a precompact conjugacy class, and since $G_{F}$ has finite index in $G,\left\{b x_{i}^{-1} b^{-1} \mid b \in G_{F}\right\}$ cannot be precompact either. It follows that, for each $i, F_{i}$ is not compact.

Now $G_{F} / K$ is locally compact abelian and contains the noncompact closed images $\bar{F}_{1}, \ldots, \bar{F}_{n}$ of $F_{1}, \ldots, F_{n}$ (the images are closed because $F_{i} K$ is closed for each $i$ ). Hence by Lemma 5.1 there is a sequence of characters of $G_{F} / K$ converging to 1 uniformly on compacta such that each character is nontrivial on $\bar{F}_{1}, \ldots, \bar{F}_{n}$. This sequence lifts to a sequence $\left\{\chi_{n}\right\}$ of one dimensional characters of $G_{F}$ converging to 1 uniformly on compacta and such that $\chi_{n} \mid F_{i}$ is nontrivial for each $n$ and $i$. It follows that for each $n$ and $i$ there is an $b \in G_{F}$ with $\chi_{n}\left(x_{i} b x_{i}^{-1} b^{-1}\right) \neq 1$. Hence $x_{i} \notin H\left(\chi_{n}\right)$, the stable group of $\chi_{n}$, for each $n$ and $i$, and so for each $n, H\left(\chi_{n}\right)=G_{F}$. The Mackey theory implies that each induced representation $U^{\chi_{n}}$ is irreducible, and by Theorem 4.4 of [7] we have that every subsequence of $U^{x_{n}}$ weakly contains $U^{1}$, the regular representation of $G / G_{F}$ lifted to $G$, and hence weakly contains every subrepresentation of $U^{1}$. This is easily seen to imply that $U^{\boldsymbol{x}_{n}}$ converges to every irreducible subrepresentation of $U^{1}$, and unless $G=G_{F}$ there will be more than one such subrepresentation. Thus if $\hat{G}$ is $T_{2}, G \in[F C]^{-}$.

5.3 Theorem. If $G \in[S I N]$, then $\hat{G}$ is Hausdorff if and only if $G$ is the projective limit of $[Z]$ groups.

Proof. Suppose $G$ is the projective limit of [Z] groups. Proposition 2.2 of [20] states that every factor representation of $G$ is actually a representation of one of the $[Z]$ groups. Thus $G$ has only finite dimensional irreducible representations. It is clear that $G \in[F C]^{-}$; therefore, by 3.6, $\hat{G}$ is Hausdorff. (See also [21].)

Suppose $\hat{G}$ is Hausdorff. Then since $G$ is $[S I N]$ and type I the corollary to 
4.6 implies that $G$ has only finite dimensional irreducible representations.

Theorem 3 of [20] states that $G$ must be the projective limit of Lie groups, each containing an open $[Z]$ subgroup of finite index. Moreover by 5.2, $G \in[F C]^{-}$. Therefore to establish 5.3 we need only prove the following lemma.

5.4 Lemma. If $G$ is an $[F C]^{-}$Lie group which contains a [Z] subgroup of finite index, then $G$ is a $[Z]$ group.

Remark. The lemma is also true if we replace "Lie" by "compactly generated", but some such hypothesis is necessary. Here is an example of an $[F C]^{-}$group which is not a $[Z]$ group but which contains a normal abelian subgroup of index 2 . Let $A=\left(\Pi_{1}^{\infty} \mathrm{Z}_{2}\right) \oplus \Sigma_{1}^{\infty} \mathrm{Z}_{2}$, where $\Pi \mathrm{Z}_{2}$ has the product topology and the weak direct sum $\Sigma Z_{2}$ has the discrete topology. Define the map $\alpha$ as follows: for $\left(z_{i}\right) \in \Pi \mathbf{Z}_{2}$ and $\left(w_{i}\right) \in \Sigma \mathbf{Z}_{2}$, put $\alpha\left(\left(z_{i}\right),\left(w_{i}\right)\right)=\left(\left(z_{i}+w_{i}\right),\left(w_{i}\right)\right)$. Then $\alpha$ is a continuous homomorphism of $A$ into itself and $\alpha^{2}=$ identity, so that $\alpha$ is a topological group automorphism. Now let $G=A \times_{\eta} \mathbf{Z}_{2}$, where $\eta(x)=\alpha^{x}$. Then one may verify that the commutator of $G$ is a subgroup of $\Pi Z_{2} \subset A$ and hence is precompact, and that $Z(G)=\Pi Z_{2}$. Thus $G$ is an $[F D]^{-}$group with an abelian subgroup of index 2 , but $G$ is not a [Z] group.

Proof of 5.4. Since open subgroups of $[Z]$ groups are $[Z]$ groups, we may assume that the $[Z]$ subgroup of finite index is normal. We first reduce 5.4 to a problem in abelian group theory. Suppose that $G$ is a finite extension of a [Z] group $H$, and that $Z(G) \cap Z(H)$ has compact quotient in $Z(H)$. Then $Z(G) \cap$ $Z(H)$ has compact quotient in $G$, and so does $Z(G)$. Therefore we may simply show $Z(G) \cap Z(H)$ has compact quotient in $Z(H)$.

Now $Z(G)$ is the set of fixed points of $\mathscr{I}(G)$, the inner automorphisms of $G$. Each element of $g(G)$ maps $H$ onto itself and hence $Z(H)$ onto itself. Moreover if $\alpha \in \mathscr{I}(G)$ corresponds to an element of $H, \alpha \mid Z(H)$ is trivial. Since $G / H$ is finite, $G$ acts as a finite group $F$ of automorphisms of $Z(H)$. We say that a topological group automorphism $\gamma$ of a locally compact group $L$ is of bounded displacement if the set $\left\{y(x) x^{-1} \mid x \in L\right\}$ is precompact (this notion was introduced by $T$ its in [28]). In the present case we assume $\dot{G}$ is $[F C]^{-}$, and so it is simple to verify that each $a \in F$ is of bounded displacement. Moreover, since $G$ is a Lie group so is the closed subgroup $Z(H)$. Therefore we are reduced to 5.5 below.

5.5 Lemma. Let $F$ be a finite group of automorphisms of the abelian Lie group A. Suppose each element of $F$ bas bounded displacement. Then the group of common fixed points of $F$ bas compact quotient in $A$.

Proof. We must reduce the problem a bit further. Since $A$ is an abelian Lie 
group, $A=T^{n} \oplus \mathbf{R}^{m} \oplus D$, where $D$ is discrete. Each a maps $T^{n} \oplus \mathbf{R}^{m}$ into itself (since $T^{n} \oplus \mathbf{R}^{m}$ is the connected component of the identity) and $T^{n}$ into itself (since $T^{n}$ is maximal compact in $T^{n} \oplus \mathrm{R}^{m}$ ). Thus a defines an automorphism $\bar{a}$ of $D$. We denote by $\bar{F}$ the finite group of such automorphisms of $D$, each of which has finite displacement. For each $\bar{\alpha} \in \bar{F}, \operatorname{ker}(\bar{\alpha}-$ id $)$ has finite index in $D$. It follows that $D^{\prime}=\bigcap_{\operatorname{ker}}(\bar{\alpha}-\mathrm{id})$ has finite index in $D$. Let $A^{\prime}=$ $T^{n} \oplus \mathbf{R}^{m} \oplus D^{\prime}$. Each a maps $A^{\prime}$ into itself; moreover $A / A^{\prime}$ is finite, so that if $\bigcap_{\text {ker }}\left(\alpha \mid A^{\prime}-\mathrm{id}\right)$ has compact quotient in $A^{\prime}, \bigcap_{\mathrm{ker}}(\alpha-\mathrm{id})$ has compact quotient in $A$. Finally, since each $\alpha \mid A^{\prime}$ is of bounded displacement and range $\left(\alpha \mid A^{\prime}-\mathrm{id}\right)$ $\subset T^{n} \oplus \mathbf{R}^{m}$, we actually have range $\left(\alpha \mid A^{\prime}-\mathrm{id}\right) \subset T^{n}$ for each $\alpha$. Thus 5.5 would follow from the following.

5.6 Lemma. Let $F$ be a finite group of automorphisms of the locally compact abelian group $A=T^{n} \oplus \mathbf{R}^{m} \oplus D$ such that the range of $(\alpha-\mathrm{id})$ lies in $T^{n}$ for each $\alpha \in F$. Then $\bigcap_{\mathrm{ker}}(\alpha-\mathrm{id})$, the group of fixed points of $F$, bas compact quotient in $A$.

Proof. We dua lize (see [13] for details on duals and adjoint maps) and obtain the following equivalent lemma, which we prove outright.

5.7 Lemma. Let $F$ be a finite group of automorphisms of the locally compact abelian group $A=\mathbf{Z}^{n} \oplus \mathbf{R}^{p} \oplus K$, where $K$ is compact. Suppose that $\operatorname{ker}(\alpha-\mathrm{id}) \supset \mathbf{R}^{p} \oplus K$ for each $\alpha \in F$. Then $\Sigma_{\alpha \in F}$ range $(\alpha-\mathrm{id})=\overline{\Sigma_{\alpha \in F} \text { range }(\alpha-\mathrm{id})}$ is discrete in the relative topology.

Proof. It is enough to show $\Sigma$ range $(\alpha-i d)$ is discrete in the relative topology. Suppose $|F|=m$. The subgroup $B=\mathbf{R}^{p} \oplus K$ is characteristic in $A$. Let $\left({ }_{b}^{z}\right)$ denote the generic element of $A$, where $z$ is a column of $n$ integers and $b \in B$, and let $\alpha_{1}, \cdots, \alpha_{m}$ be the elements of $F$. Since each $\alpha_{i} \in F$ is the identity on $B$, we have

$$
a_{i}\left(\begin{array}{l}
z \\
b
\end{array}\right)=\left(\begin{array}{c}
M_{i} z \\
Y_{i} z+b
\end{array}\right)
$$

where each $M_{i}$ is a $n \times n$ integer valued matrix and each $Y_{i}$ is a $n$-tuple of elements of $B$. We may therefore write each

we then have

$$
\alpha_{i}=\left(\begin{array}{cc}
M_{i} & 0 \\
Y_{i} & 1
\end{array}\right) ;
$$

$$
a_{i} \alpha_{j}=\left(\begin{array}{cc}
M_{i} M_{j} & 0 \\
Y_{i} M_{j}+Y_{j} & 1
\end{array}\right),
$$


and since $\alpha_{i} \alpha_{i}^{-1}=\mathrm{id}, M_{i}$ is invertible and in fact $M_{i} \in \mathrm{SL}^{ \pm}(n, Z)$ for each $i$; that is, $M_{i}$ is an automorphism of $Z^{n}$ for each $i$. If we define the integer $k_{i j}$ by $\alpha_{k_{i j}}=\alpha_{i} \alpha_{j}$ we have

(1) $M_{i} M_{j}=M_{k_{i j}}$ and

(2) $Y_{k_{i j}}=Y_{i} M_{j}+Y_{j}$.

Now

$$
\operatorname{range}\left(\alpha_{i}-\mathrm{id}\right)=\operatorname{range}\left(\begin{array}{cc}
M_{i}-I & 0 \\
Y_{i} & 0
\end{array}\right)=\left\{\left(\begin{array}{c}
M_{i} z-z \\
Y_{i} z
\end{array}\right) \mid z \in \mathbf{Z}^{n}\right\},
$$

and

$$
\sum_{\text {range }}\left(\alpha_{i}-\mathrm{id}\right)=\left\{\sum_{i=1}^{m}\left(\begin{array}{c}
M_{i} z_{i}-z_{i} \\
Y_{i} z_{i}
\end{array}\right) \mid z_{i} \in \mathbf{Z}^{n} \text { each } i\right\}
$$

Since $B$ is open in $Z^{n} \oplus B$, it would suffice to show $B \cap \Sigma$ range $\left(\alpha_{i}-\right.$ id $)$ is finite. Let us therefore observe that

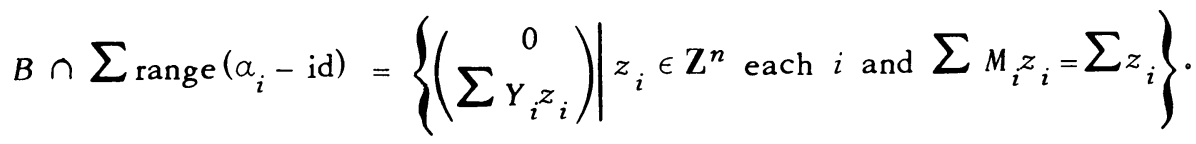

We point out that $B \cap \Sigma_{\text {range }}\left(\alpha_{i}-i d\right)$ is finitely generated, as a subgroup of the abelian subgroup of $B$ generated by the coordinate entries of $Y_{1}, \cdots, Y_{m}$. Next we show that $B \cap \Sigma_{\text {range }}\left(\alpha_{i}-i d\right)$ is torsion. In fact by (2) we have $Y_{j} z_{j}=Y_{k_{i j}} z_{j}-Y_{i} M_{j} z_{j}$ for each $i$ and $j$ and any $z_{j} \in Z^{n}$. It follows that if $\Sigma Y_{j} z_{j} \in B \cap \Sigma$ range $\left(\alpha_{i}-\right.$ id) (i.e., if $\left.\Sigma M_{j} z_{j}=\Sigma z_{j}\right)$ we have

$$
\begin{aligned}
\sum_{j=1}^{m} Y_{j} z_{j} & =\sum_{j=1}^{m} Y_{k_{i j}} z_{j}-Y_{i}\left(\sum_{j=1}^{m} M_{j} z_{j}\right)=\sum_{j=1}^{m} Y_{k_{i j}} z_{j}-Y_{i} \sum_{j=1}^{m} z_{j} \\
& =\sum_{j=1}^{m}\left(Y_{k_{i j}}-Y_{i}\right)_{j} \text { for each } i_{0}
\end{aligned}
$$

But then

$$
m \sum_{j=1}^{m} Y_{j} z_{j}=\sum_{i=1}^{m} \sum_{j=1}^{m}\left(Y_{k_{i j}}-Y_{i}\right) z_{j}=\sum_{j=1}^{m}\left(\sum_{i=1}^{m}\left(Y_{k_{i j}}-Y_{i}\right)\right) z_{j}=0
$$

since $k_{i j}$ runs over $1, \ldots, m$ as $i$ does whenever $j$ is fixed. Thus $B \cap$ $\Sigma$ range $\left(\alpha_{i}-i d\right)$ is finite; it follows that $\Sigma$ range $\left(\alpha_{i}-\right.$ id $)$ is discrete, and Lemmas 5.7 and 5.4 are proved.

6. $[I N]$ groups with discrete duals. We conclude this paper by describing the second countable $[I N]$ groups with discrete dual spaces. As noted in the introduction, Baggett [2] has shown that any second countable group with 
discrete dual is compact; here we sketch a simple proof of this fact in the [IN] case.

The first step is to observe that a separable $C^{*}$ algebra $A$ with discrete dual is a dual $C^{*}$ algebra; that is, has the following form: For each $\alpha \in \hat{A}$, let $I_{a}$ be the unique closed two sided ideal of $A$ whose dual space is $\{a\}$ (see 3.2 of [5].) Then since $I_{a}$ is a separable $C^{*}$ algebra with only one irreducible * representation, $I_{\alpha}$ is isomorphic to the algebra of all compact operators on a separable Hilbert space (see p. 296 of [22]). Let $B$ consist of all sequences $\left\{x_{a}\right\}$ such that $x_{a} \in I_{a}$ for each $\alpha$ and $\left\|x_{a}\right\| \rightarrow 0$ as $\alpha \rightarrow \infty$. Define algebraic operations and involution componentwise, and define $\left\|\left\{x_{a}\right\}\right\|=\sup \left\|x_{a}\right\|$. Then $B$ is a $C^{*}$ algebra, and using the elementary theory of $C^{*}$ algebras, especially the material in 3.2 of [5], one can show readily that $A$ is isomorphic to $B$ as a $C^{*}$ algebra. It also follows easily that every irreducible ${ }^{*}$ representation of $A$ is trivial on every $I_{a}$ expect one, and there it is faithful. Further any ${ }^{*}$ representation of $A$ decomposes into the discrete direct sum of these irreducible ${ }^{*}$ representations.

Now consider a second countable $[I N]$ group $G$ with discrete dual. Then $G$ is type $I[I N]$ and therefore a finite extension of $G_{F}$, an $[F C]^{-}$group. Now $G_{F}$ is the directed union of open compactly generated subgroups, each of which is $[F D]^{-}$by 3.20 of [12]. The class of locally compact amenable groups is closed under extension and directed unions (see e.g., Greenleaf, Invariant means on topological groups and their applications) and so $G$ must be amenable. Therefore the regular representation of $G$ is faithful on $C^{*}(G)$ and hence contains the trivial one dimensional representation as a direct summand. This means that $L^{2}(G)$ has a one dimensional translation invariant subspace, and it follows that $G$ is compact.

\section{REFERENCES}

1. L. Auslander and C. C. Moore, Unitary representations of solvable Lie groups, Mem. Amer. Math. Soc. No. 62 (1966), 199 pp. MR 34 \#7723.

2. L. Baggett, A separable group having a discrete dual space is compact (preprint).

3. R. J. Blattner, Positive definite measures, Proc. Amer. Math. Soc. 14 (1963), 423-428. MR $26 \# 5095$.

4. J. Braconnier, Sur les groups topologiques localement compacts, J. Math. Pures Appl. (9) 27 (1948), 1-85. MR 10, 11.

5. J. Dixmier, Les $C^{*}$-algèbres et leurs représentations, Cahiers Scientifiques, fasc. 29, Gauthier-Villars, Paris, 1964. MR 30 \#1404.

6. J. M. G. Fell, The dual spaces of $C^{*}$ algebras, Trans. Amer. Math. Soc. 94 (1960), 365-403. MR $26 \# 4201$.

7. - Weak containment and induced representations of groups, Canad. J. Math. 14 (1962), 237-268. MR 27 \#242.

8. J. G. Glimm, Locally compact transformation groups, Trans. Amer. Math. Soc. 101 (1961), 124-138. MR $25 \# 146$.

9. V. M. Gluškov, The structure of locally compact groups and Hilbert's fifth problem, Uspehi Mat. Nauk 12 (1957), no. 2 (74), 3-41; English transl., Amer. Math. Soc. Transl. (2) 15 (1960), 55-93. MR 21 \#698; MR $22 \# 5690$. 
10. S. Grosser and M. Moskowitz, On central topological groups, Trans. Amer. Math. Soc. 127 (1967), 317-340. MR 35 \#292.

11. - Representation theory of central topological groups, Trans. Amer. Math. Soc. 129 (1967), 361-390. MR 37 \#5327.

12. Compactness conditions in topological groups, J. Reine. Angew Math. 246 (1971), $1-40$.

13. E. Hewitt and K. A. Ross, Abstract harmonic analysis. Vol. I: Structure of topological groups. Integration theory, group representations, Die Grundlehren der math. Wissenschaften, Band 115, Academic Press, New York; Springer-Verlag, Berlin, 1963. MR $28 \# 158$.

14. E. Kaniuth, Die Struktur der regulären Darstellung lokalkompakter Gruppen mit invarianter Umgebungsbasis der Eine (preprint).

15. J. L. Kelley, General topology, Van Nostrand, Princeton, N.J., 1955. MR 16, 1136.

16. A. Kleppner, Multipliers on abelian groups, Math. Ann. 158 (1965), 11-34. MR 30 \#856.

17. G. W. Mackey, Induced representations of locally compact groups. I, Ann. of Math. (2) 55 (1952), 101-139. MR 13, 434.

18. - The theory of group representations, Lecture Notes (Summer, 1955), 3 vols., University of Chicago, Dept. of Math., Chicago, Ill., 1955. MR 19, 117.

19. Unitary representations of group extensions. I, Acta Math. 99 (1958), 265-311. MR $20 \# 4789$.

20. C. C. Moore, Groups with finite dimensional irreducible representations (preprint).

21. R. Mosak, The $L^{1}$ and $C^{*}$ algebras of $[F I A]_{B}^{-}$groups and their representations, Trans. Amer. Math. Soc. 163 (1972), 277-310。

22. M. A. Nałmark, Normed rings, GITTL, Moscow, 1956; English transl., rev. ed., Noordhoff, Groningen, 1964. MR 19, 870; MR 34 \#4928.

23. B. H. Neumann, Groups with finite classes of conjugate elements, Proc. London Math. Soc. (3) 1 (1951), 178-187. MR 13, 316.

24. R. Phelps, Lectures on Choquet's theorem, Van Nostrand, Princeton, N.J., 1966. MR 33 \#1690.

25. L. C. Robertson, A note on the structure of Moore groups, Bull. Amer. Math. Soc. 75 (1969), 594-599. MR 39 \#7027.

26. I. Schochetman, Topology and the duals of certain locally compact groups, Trans. Amer. Math. Soc. 150 (1970), 477-489. MR 42 \#422.

27. E. Thoma, Über unitäre Darstellungen abzählbares, diskreter Gruppen, Math. Ann. 153 (1964), 111-138. MR 28 \#3332. 107.

28. J. Tits, Automorphisms à déplacement borné, Topology 3 (1964), suppl. 1, 97-

29. D. M. Lee and T. S. Wu, On existence of compact open normal subgroups of 0dimensional groups, Proc. Amer. Math. Soc. 26 (1970), 265-528. 70118

DEPARTMENT OF MATHEMATICS, TULANE UNIVERSITY, NEW ORLEANS, LOUISIANA 